\title{
Applications of Adaptive Filtering: Recent Advancements in Active Noise Control
}

\author{
Akhtar Muhammad Tahir ${ }^{1}$, Mitsuhashi Wataru ${ }^{2}$ and Nishihara Akinori ${ }^{3}$ \\ ${ }^{1,2}$ The University of Electro-Communications, Tokyo \\ ${ }^{3}$ Tokyo Institute of Technology, Tokyo \\ Japan
}

\section{Introduction}

ACTIVE NOISE CONTROL (ANC) is based on the simple principle of destructive interference of propagating acoustic waves, (Elliot, 2001). The basic idea was proposed in 1936 (Lueg, 1936), however, real applications were quite limited till recently. Thanks to advancement in the algorithms for adaptive signal processing and their implementation using digital signal processors (DSPs); many successful applications of ANC have been reported, the most famous being noise reduction headsets (Gan \& Kuo, 2002; Kuo et al., 2006).

The most popular adaptive algorithm used for ANC applications is the filtered-x least mean square (FxLMS) algorithm (Kuo \& Morgan, 1996) which is a modified version of the LMS algorithm (Widrow \& Stearns, 1985). The FxLMS algorithm is computationally simple, but its convergence speed is slow. Different ANC algorithms, with improved convergence properties, have been proposed, viz., 1) lattice-ANC systems (Park \& Sommerfeldt, 1996); 2) infinite impulse response (IIR) filter-based LMS algorithms called filtered-u recursive LMS (FuRLMS) (Eriksson et al., 1987), and filtered-v algorithms (Crawford \& Stewart, 1997); 3) recursive least squares (RLS) based algorithms called filtered-x RLS (FxRLS) (Kuo \& Morgan, 1996) and filtered-x fast-transversal-filter (FxFTF) (Bouchard \& Quednau, 2000); and 4) frequency-domain-ANC systems (see (Kuo \& Tahernezhadi, 1997) and references there in). There are the following problems with these approaches: 1) IIR-based structures have inherent stability problems; 2) other approaches mentioned above increase the computational burden substantially; and 3) RLS-based ANC systems have numerical instability problems. These reasons make FxLMS still a good choice for ANC applications, and hence, in this chapter we describe various concepts and methods using FxLMS algorithm.

The main objective of this chapter is to provide a comprehensive review of adaptive filtering algorithms developed and employed for ANC systems. We also provide some recent results for two challenging problems: ANC of impulsive-like noise sources, and mitigating effect of the uncorrelated disturbances for which a correlated reference signal is not available. We see that simple modifications and extensions of the existing algorithms and methods improve robustness of the ANC systems.

The outline of the chapter is as follows. Section 2 details FxLMS algorithm for feedforward and feedback type ANC systems. It also highlights signal processing issues and open problems for further research. Section 3 describes development of various adaptive algorithm for ANC for Impulsive Noise Sources, and Section 4 addresses issue of Mitigating 


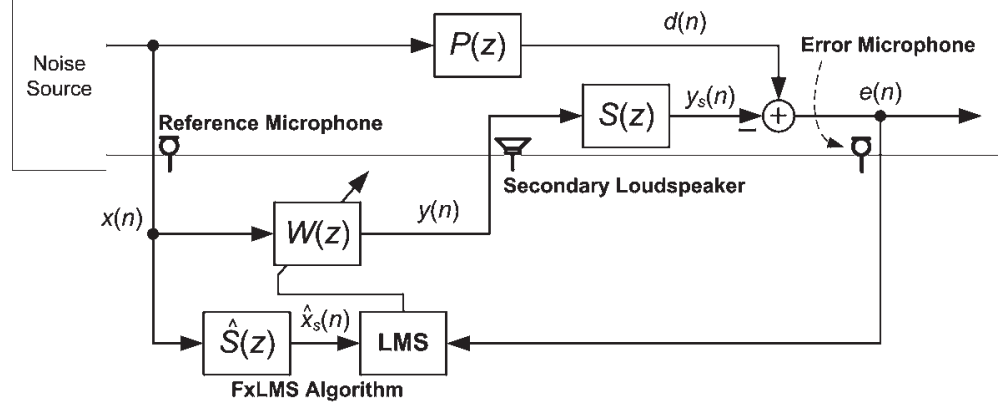

Fig. 1. Block diagram of FxLMS algorithm-based single-channel feedforward ANC system.

Uncorrelated Disturbance appearing the error microphone of feedforward ANC system. Section 5 presents results of Computer Simulations for two case studies discussed in this chapter, viz., ANC for impulsive sources, and mitigating effect of uncorrelated disturbance. Section 6 is an An Outlook on Recent ANC Applications and Section 7 gives the Concluding Remarks.

\section{FxLMS algorithm}

In this section we give description of FxLMS algorithm for single-channel feedforward and feedback type ANC systems. Furthermore, a brief review on various signal processing issues, solved and unsolved, is also detailed.

\subsection{Feedforward ANC}

The block diagram for a single-channel feedforward ANC system using the FxLMS algorithm is shown in Fig. 1, where $P(z)$ is primary acoustic path between the reference noise source and the error microphone. The reference noise signal $x(n)$ is filtered through $P(z)$ and appears as a primary noise signal at the error microphone. The objective of the adaptive filter $W(z)$ is to generate an appropriate antinoise signal $y(n)$ propagated by the secondary loudspeaker. This antinoise signal combines with the primary noise signal to create a zone of silence in the vicinity of the error microphone. The error microphone measures the residual noise $e(n)$, which is used by $W(z)$ for its adaptation to minimize the sound pressure at error microphone. Here $\hat{S}(z)$ accounts for the model of the secondary path $S(z)$ between the output $y(n)$ of the controller and the output $e(n)$ of the error microphone. The filtering of the reference signal $x(n)$ through $\hat{S}(z)$ is demanded by the fact that the output $y(n)$ of the adaptive filter is filtered through $S(z)$ (Kuo \& Morgan, 1996).

Assuming that $W(z)$ is an FIR filter of tap-weight length $L_{w}$, the secondary signal $y(n)$ is expressed as

$$
y(n)=\boldsymbol{w}^{T}(n) \boldsymbol{x}(n) .
$$

where

$$
\boldsymbol{w}(n)=\left[w_{0}(n), w_{1}(n), \cdots, w_{L_{w}-1}(n)\right]^{T}
$$

is the tap-weight vector, and

$$
\boldsymbol{x}(n)=\left[x(n), x(n-1), \cdots, x\left(n-L_{w}+1\right)\right]^{T}
$$




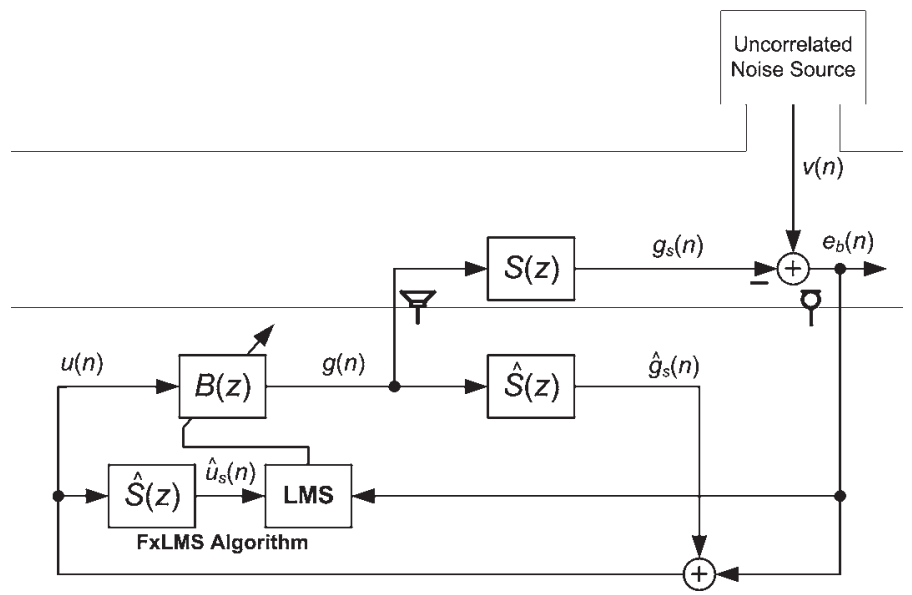

Fig. 2. Block diagram of FxLMS algorithm-based single-channel feedback ANC systems.

is an $L_{w}$-sample vector the reference signal $x(n)$. The residual error signal $e(n)$ is given as

$$
e(n)=d(n)-y_{s}(n)
$$

where $d(n)=p(n) * x(n)$ is the primary disturbance signal, $y_{s}(n)=s(n) * y(n)$ is the secondary canceling signal, $*$ denotes linear convolution, and $p(n)$ and $s(n)$ are impulse responses of the primary path $P(z)$ and secondary path $S(z)$, respectively.

Minimizing the mean squared error (MSE) cost function; $J(n)=E\left\{e^{2}(n)\right\} \approx e^{2}(n)$, where $E\{\cdot\}$ is the expectation of quantity inside; the FxLMS update equation for the coefficients of $W(z)$ is given as

$$
\boldsymbol{w}(n+1)=\boldsymbol{w}(n)+\mu_{w} e(n) \hat{\boldsymbol{x}}_{s}(n)
$$

where $\mu_{w}$ is the step size parameter,

$$
\hat{\boldsymbol{x}}_{s}(n)=\left[\hat{x}_{s}(n), \hat{x}_{s}(n-1), \cdots, \hat{x}_{s}\left(n-L_{w}+1\right)\right]^{T}
$$

is filtered-reference signal vector being generated as

$$
\hat{\boldsymbol{x}}_{s}(n)=\hat{s}(n) * \boldsymbol{x}(n),
$$

where $\hat{s}(n)$ is impulse response of the secondary path modeling filter $\hat{S}(z)$.

\subsection{Feedback ANC}

The feedforward strategy as described above is widely used in ANC systems, where an independent reference signal $x(n)$ is available and is well correlated with the primary noise $d(n)$. Whenever the reference signal related to the primary noise source is unavailable or several reference signals are in the enclosure, the use of feedforward control becomes impractical. Under such circumstances, feedback control may be envisaged, in which measured residual error signals are used to derive the secondary sources. The block diagram for feedback ANC system is shown in Fig. 2, where $v(n)$ represents a noise source for which a correlated reference signal is not available. As shown, the feedback ANC system comprises 
only error microphone and secondary loudspeaker. The output $g(n)$ of the feedback ANC $B(z)$ passes through $S(z)$ to generate the residual error signal $e_{b}(n)$ as

$$
e_{b}(n)=v(n)-g_{s}(n),
$$

where $g_{s}(n)=s(n) * g(n)$ is the cancelling signal for $v(n)$. The residual error signal $e_{b}(n)$ is picked by the error microphone and is used in the adaptation of the FxLMS algorithm for $B(z)$. The reference signal for $B(z)$ is internally generated by filtering $g(n)$ through secondary path model $\hat{S}(z)$ and adding it to the residual error signal $e_{b}(n)$ as

$$
u(n)=e_{b}(n)+\hat{g}_{S}(n)=\left[v(n)-g_{s}(n)\right]+\hat{g}_{S}(n),
$$

where $\hat{g}_{s}(n)=\hat{s}(n) * g(n)$ is the estimate of cancelling signal $g_{s}(n)$. Assuming that the secondary path is perfectly identified; which can be obtained by using offline (Kuo \& Morgan, 1996) and/or online modeling techniques (Akhtar et al., 2005; 2006); $\hat{g}_{s}(n) \approx g_{s}(n)$, and hence Eq. (9) simplifies to give estimate of uncorrelated noise source as $u(n) \rightarrow v(n)$. Using this internally generated reference signal ${ }^{1}$, the output $g(n)$ of feedback ANC $B(z)$ is computed as

$$
g(n)=\boldsymbol{b}^{T}(n) \boldsymbol{u}(n) .
$$

where

$$
\boldsymbol{b}(n)=\left[b_{0}(n), b_{1}(n), \cdots, b_{L_{b}-1}(n)\right]^{T}
$$

is the tap-weight vector for $B(z)$,

$$
\boldsymbol{u}(n)=\left[u(n), u(n-1), \cdots, u\left(n-L_{b}+1\right)\right]^{T}
$$

is the corresponding reference signal vector for $u(n)$, and $L_{b}$ is the tap-weight length of $B(z)$. Finally the FxLMS algorithm for updating $B(z)$ is given as

$$
\boldsymbol{b}(n+1)=\boldsymbol{b}(n)+\mu_{b} e_{b}(n) \hat{\boldsymbol{u}}_{s}(n)
$$

where $\mu_{b}$ is the step size parameter for $B(z)$, and filtered-reference signal vector $\hat{\boldsymbol{u}}_{s}(n)=$ $\left[\hat{u}_{s}(n), \hat{u}_{s}(n-1), \cdots, \hat{u}_{s}\left(n-L_{b}+1\right)\right]^{T}$ is generated as

$$
\hat{\boldsymbol{u}}_{s}(n)=\hat{s}(n) * \boldsymbol{u}(n) .
$$

In feedback ANC, hence, the basic idea is to estimate the primary noise $v(n)$, and use it as a reference signal $u(n)$ for the feedback ANC filter $B(z)$. It is worth mentioning that the feedforward ANC provides wider control bandwidth within moderate controller gain than the feedback ANC, whereas feedback ANC gives significant performance for narrowband or predictable noise sources.

\subsection{Review on signal processing challenges}

The FxLMS algorithm appears to be very tolerant of errors made in the modeling of $S(z)$ by the filter $\hat{S}(z)$. As shown in (Elliott et. al., 1987; Morgan, 1980), with in the limit of slow

\footnotetext{
${ }^{1}$ This is why FxLMS algorithm for feedback ANC systems is sometimes referred as internal model control (Kuo \& Morgan, 1996)
} 
adaptation, the algorithm will converge with nearly $90^{\circ}$ of phase error between $\hat{S}(z)$ and $S(z)$. Therefore, offline modeling can be used to estimate $S(z)$ during an initial training stage for ANC applications (Kuo \& Morgan, 1999). For some applications, however, the secondary path may be time varying, and it is desirable to estimate the secondary path online when the ANC is in operation (Saito \& Sone, 1996).

There are two different approaches for online secondary path modeling. The first approach, involving the injection of additional random noise into the ANC system, utilizes a system identification method to model the secondary path. The second approach attempts to model it from the output of the ANC controller, thus avoiding the injection of additional random noise into the ANC system. A detailed comparison of these two online modeling approaches can be found in (Bao et al., 1993a), which concludes that the first approach is superior to the second approach on convergence rate, speed of response to changes of primary noise, updating duration, computational complexities, etc.

The basic additive random noise technique for online secondary path modeling in ANC systems is proposed by (Eriksson \& Allie, 1989). This ANC system comprises two adaptive filters; FxLMS algorithm based noise control filter $W(z)$, and LMS algorithm based secondary path modeling filter $\hat{S}(z)$. Improvements in the Eriksson's method have been proposed in (Bao et al., 1993b; Kuo \& Vijayan, 1997; Zhang et al., 2001). These improved methods introduce another adaptive filter into the ANC system of (Eriksson \& Allie, 1989), which results in increased computational complexity. The methods proposed in (Akhtar et al., 2005; 2006) suggest modifications to Eriksson's method such that improved performance is realized without introducing a third adaptive filter. The development of robust and efficient online secondary path modeling algorithm, without requiring additive random noise, is critical and demands further research.

The feedforward ANC system shown in Fig. 1 uses the reference microphone to pick up the reference noise $x(n)$, processes this input with an adaptive filter to generate an antinoise $y(n)$ to cancel primary noise acoustically in the duct, and uses an error microphone to measure the error $e(n)$ and to update the adaptive filter coefficients. Unfortunately, a loudspeaker on a duct wall will generate the antinoise signal propagating both upstream and downstream. Therefore, the antinoise output to the loudspeaker not only cancels noise downstream, but also radiates upstream to the reference microphone, resulting in a corrupted reference signal $x(n)$. This coupling of acoustic waves from secondary loudspeaker to the reference microphone is called acoustic feedback. One simple approach to neutralize the effect of acoustic feedback is to use a separate feedback path modeling filter with in the controller. This electrical model of the feedback path is driven by the antinoise signal, $y(n)$, and its output is subtracted from the reference sensor signal, $x(n)$. The feedback path modeling filter may be obtained offline prior to the operation of ANC system when the reference noise $x(n)$ does not exist. In many practical cases, however, $x(n)$ always exists, and feedback may be time varying as well. For these cases, online modeling of feedback path is needed to ensure the convergence and stability of the FxLMS algorithm for ANC systems. For a detailed review on existing signal processing methods and various other techniques for feedback neutralization in ANC systems, the reader is referred to (Akhtar et al., 2007) and references there in.

In the case of narrowband noise sources with signal energy being concentrated at a few representative harmonics, the reference microphone in Fig. 1 can be replaced with a non-acoustic sensor, e.g., a tachometer in the case rotating machines. The output from non-acoustic sensor is used to internally generate the reference signal, which may be an impulse train with a period equal to the inverse of the fundamental frequency of periodic 


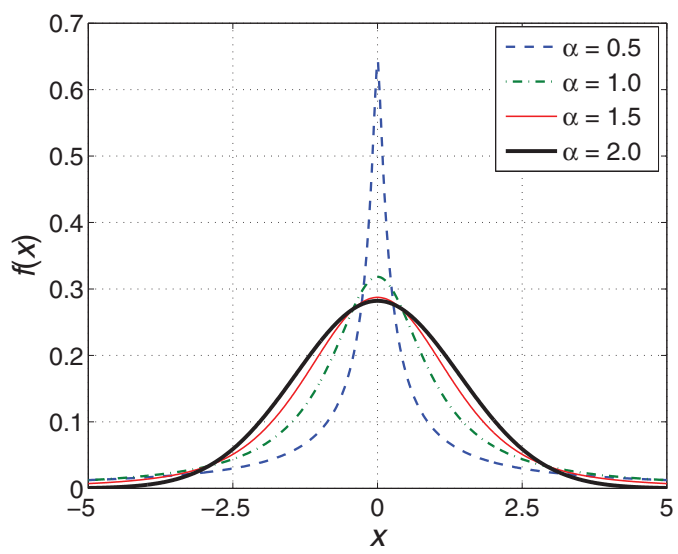

Fig. 3. The PDFs of standard symmetric $\alpha$-stable $(S \alpha S)$ process for various values of $\alpha$.

noise, or sinusoids that have the same frequencies as the corresponding harmonic components (Kuo \& Morgan, 1996). Essentially, a narrowband ANC system would assume the reference signal $x(n)$ has the same frequency as the primary noise $d(n)$ at the error microphone. In many practical situations, the reference sinusoidal frequencies used by the adaptive filter may be different than the actual frequencies of primary noise. This difference is referred to as frequency mismatch (FM), and will degrades the performance of ANC systems. The effects of FM and solution to the problems have been recently studied in (Jeon et al., 2010; Kuo \& Puvvala, 2006; Xiao et al., 2005; 2006).

Another signal processing challenge is ANC for sources with nonlinear behavior. It has been demonstrated that the FxLMS algorithm gives very poor performance in the case of nonlinear processes (Strauch \& Mulgrew, 1998). For efficient algorithms for ANC of non linear source, see (Reddy et al., 2008) and references there in.

In many practical situations, it is desirable to shift the quiet zone away from the location of error microphones to a virtual location where error microphone cannot be installed (Bonito et al., 1997). One interesting example is recently investigated snore ANC system, where headboard of bed is mounted with loudspeakers and microphones (Kuo et al., 2008). In this case, the error microphone cannot be placed at the ears of the bed partner, where maximum cancellation is required, and hence an efficient virtual sensing technique is required to improve the noise reduction around ears using error microphones installed on the headboard. There has been a very little research on active control of moving noise sources. It is obvious that acoustic paths will be highly time varying in such cases, and hence the optimal solution for ANC would also vary when the positions of primary noise source change (Guo \& Pan, 2000). The behavior of adaptive filters for ANC of moving noise sources is studied in (Omoto et al., 2002), and further researcher is needed to investigate the effects of time varying paths and developing efficient control algorithms that can cope with the Doppler effects.

In the following sections we discuss challenging task of ANC for impulsive noise sources, and mitigating effect of uncorrelated disturbance. We demonstrate that proposed algorithms and methods can greatly improve the convergence and performance of ANC systems for these tasks. 


\section{ANC for impulsive noise sources}

There are many important ANC applications that involve impulsive noise sources (Kuo et al., 2010). In practice, the impulsive noises are often due to the occurrence of noise disturbance with low probability but large amplitude. There has been a very little research on active control of impulsive noise, at least up to the best knowledge of authors. In practice the impulsive noises do exist and it is of great meaning to study its control.

An impulsive noise can be modeled by stable non-Gaussian distribution (Nikias, 1995; Shao $\&$ Nikias, 1993). We consider impulse noise with symmetric $\alpha$-stable $(\mathrm{S} \alpha \mathrm{S})$ distribution $f(x)$ having characteristic function of the form (Shao \& Nikias, 1993)

$$
\varphi(t)=e^{j a t-\gamma|t|^{\alpha}}
$$

where $0<\alpha<2$ is the characteristics exponent, $\gamma>0$ is the scale parameter called as dispersion, and $a$ is the location parameter. The characteristics exponent $\alpha$ is a shape parameter, and it measures the "thickness" of the tails of the density function. If a stable random variable has a small value for $\alpha$, then distribution has a very heavy tail, i.e., it is more likely to observe values of random variable which are far from its central location. For $\alpha=2$ the relevant stable distribution is Gaussian, and for $\alpha=1$ it is the Cauchy distribution. An $\mathrm{S} \alpha \mathrm{S}$ distribution is called standard if $\gamma=1, a=0$. In this paper, we consider ANC of impulsive noise with standard $S \alpha S$ distribution, i.e., $0<\alpha<2, \gamma=1$, and $a=0$. The PDFs of standard $\mathrm{S} \alpha \mathrm{S}$ process for various values of $\alpha$ are shown in Fig. 3. It is evident that for small value of $\alpha$, the process has a peaky and heavy tailed distribution.

In order to improve the robustness of adaptive algorithms for processes having PDFs with heavy tails (i.e. signals with outliers), one of the following solution may be adopted:

1. A robust optimization criterion may be used to derive the adaptive algorithm.

2. The large amplitude samples may be ignored.

3. The large amplitude samples may be replaced by an appropriate threshold value.

The existing algorithms for ANC of impulsive noise are based on the first two approaches. In the proposed algorithms, we consider combining these approaches as well as borrow concept of the normalized step size, as explained later in this section. The discussion presented is with respect to feedforward ANC of Fig. 1, where noise source is assumed to be of impulse type. It is important to note that the feedback type ANC works as a predictor and hence cannot be employed for such types of sources.

\subsection{Variants of FxLMS algorithm}

Consider feedforward ANC system of Fig. 1, where we assume that noise source is impulsive and follows $S \alpha S$ distribution as explained earlier. The reference signal vector; used in the update equation of the FxLMS algorithm and in generating the cancelling signal $y(n)$; is given in Eq. (3) which shows that the samples of the reference signal $x(n)$ at different time are treated "equally". It may cause the FxLMS algorithm to become unstable in the presence of impulsive noise. To overcome this problem, a simple modification to FxLMS algorithm is proposed in (Sun et al., 2006). In this algorithm, hereafter referred as Sun's algorithm, the samples of the reference signal $x(n)$ are ignored, if their magnitude is above a certain threshold set by 
statistics of the signal (Sun et al., 2006). Thus the reference signal is modified as

$$
x^{\prime}(n)=\left\{\begin{array}{cl}
x(n), & \text { if } x(n) \in\left[c_{1}, c_{2}\right] \\
0, & \text { otherwise }
\end{array}\right.
$$

Here, the thresholding parameters $c_{1}$ and $c_{2}$ can be obtained offline for ANC systems. A few comments on choosing these parameters are given later. Thus Sun's algorithm for ANC of impulsive noise is given as (Sun et al., 2006)

$$
\boldsymbol{w}(n+1)=\boldsymbol{w}(n)+\mu_{w} e(n) \hat{\boldsymbol{x}}_{s}^{\prime}(n),
$$

where $\hat{\boldsymbol{x}}_{s}^{\prime}(n)=\left[\hat{x}_{s}^{\prime}(n), \hat{x}_{s}^{\prime}(n-1), \cdots, \hat{x}_{s}^{\prime}\left(n-L_{w}+1\right)\right]^{T}$ is generated as

$$
\hat{\boldsymbol{x}}_{s}^{\prime}(n)=\hat{s}(n) * \boldsymbol{x}^{\prime}(n),
$$

where

$$
\boldsymbol{x}^{\prime}(n)=\left[x^{\prime}(n), x^{\prime}(n-1), \cdots, x^{\prime}\left(n-L_{w}+1\right)\right]^{T}
$$

is a modified reference signal vector with $x^{\prime}(n)$ being obtained using Eq. (16). The main advantage is that the computational complexity of this algorithm is same as that of the FxLMS algorithm.

In our experience, however, Sun's algorithm becomes unstable for $\alpha<1.5$, when the PDF is peaky and the reference noise is highly impulsive. Furthermore, the convergence speed of this algorithm is very slow. The main problem is that ignoring the peaky samples in the update of FxLMS algorithm does not mean that these samples will not appear in the residual error $e(n)$. The residual error may still be peaky, and in the worst case the algorithm may become unstable. In order to improve the stability of the Sun's algorithm, the idea of Eq. (16) is extended to the error signal $e(n)$ as well, and a new error signal is obtained as (Akhtar \& Mitsuhashi, 2009a)

$$
e^{\prime}(n)= \begin{cases}e(n), & \text { if } e(n) \in\left[c_{1}, c_{2}\right] \\ 0, & \text { otherwise }\end{cases}
$$

Effectively, the idea is to freeze the adaptation of $W(z)$ when a large amplitude is detected in the error signal $e(n)$. Thus modified-Sun's algorithm for ANC of impulse noise is proposed as

$$
\boldsymbol{w}(n+1)=\boldsymbol{w}(n)+\mu_{w} e^{\prime}(n) \hat{\boldsymbol{x}}_{s}^{\prime}(n) .
$$

In order to further improve the robustness of the Sun's algorithm; instead of ignoring the large amplitude sample; we may clip the sample by a threshold value, and thus the reference signal is modified as

$$
x^{\prime \prime}(n)=\left\{\begin{array}{cc}
c_{1}, & x(n) \leq c_{1} \\
c_{2}, & x(n) \geq c_{2} \\
x(n), & \text { otherwise }
\end{array}\right.
$$

As stated earlier, ignoring (or even clipping) the peaky samples in the update of FxLMS algorithm does not mean that peaky samples will not appear in the residual error $e(n)$. The residual error may still be so peaky, that in the worst case might cause ANC to become unstable. We extend the idea of Eq. (22) to the error signal $e(n)$ as well, and a new error 
signal is obtained as

$$
e^{\prime \prime}(n)=\left\{\begin{array}{cl}
c_{1}, & e(n) \leq c_{1} \\
c_{2}, & e(n) \geq c_{2} \\
e(n), & \text { otherwise }
\end{array},\right.
$$

and hence proposed modified FxLMS (MFxLMS) algorithm for ANC of impulsive noise sources is as given below

$$
\boldsymbol{w}(n+1)=\boldsymbol{w}(n)+\mu_{w} e^{\prime \prime}(n) \hat{\boldsymbol{x}}_{s}^{\prime \prime}(n),
$$

where $\hat{\boldsymbol{x}}_{s}^{\prime \prime}(n)=\left[\hat{x}_{s}^{\prime \prime}(n), \hat{x}_{s}^{\prime \prime}(n-1), \cdots, \hat{x}_{s}^{\prime \prime}\left(n-L_{w}+1\right)\right]^{T}$ is generated as

$$
\hat{\boldsymbol{x}}_{s}^{\prime \prime}(n)=\hat{s}(n) * \boldsymbol{x}^{\prime \prime}(n),
$$

where

$$
x^{\prime \prime}(n)=\left[x^{\prime \prime}(n), x^{\prime \prime}(n-1), \cdots, x^{\prime \prime}\left(n-L_{w}+1\right)\right]^{T}
$$

is a modified reference signal vector with $x^{\prime \prime}(n)$ being obtained using Eq. (22).

It is worth mentioning that all algorithms discussed so far; Sun's algorithm (Sun et al., 2006) and its variants; require an appropriate selection of the thresholding parameters $\left[c_{1}, c_{2}\right]$. As stated earlier, the basic idea of Sun's algorithm is to ignore the samples of the reference signal $x(n)$ beyond certain threshold $\left[c_{1}, c_{2}\right]$ set by the statistics of the signal (Sun et al., 2006). Here the probability of the sample less than $c_{1}$ or larger than $c_{2}$ are assumed to be 0 , which is consistent with the fact that the tail of PDF for practical noise always tends to 0 when the noise value is approaching $\pm \infty$. Effectively, Sun's algorithm assumes the same PDF for $x^{\prime}(n)$ (see Eq. (16)) with in $\left[c_{1}, c_{2}\right]$ as that of $x(n)$, and neglects the tail beyond $\left[c_{1}, c_{2}\right]$. The stability of Sun's algorithms depends heavily on appropriate choice of $\left[c_{1}, c_{2}\right]$. We have extended this idea, that instead of ignoring, the peaky samples are replaced by the thresholding values $c_{1}$ and $c_{2}$. Effectively, this algorithm adds a saturation nonlinearity in the reference and error signal paths. Thus, the performance of this algorithm also depends on the parameters $c_{1}$ and $c_{2}$.

In order to overcome this difficulty of choosing appropriate thresholding parameters, we propose an FxLMS algorithm that does not use modified reference and/or error signals, and hence does not require selection of the thresholding parameters $\left[c_{1}, c_{2}\right]$. Following the concept of normalized LMS (NLMS) algorithm (Douglas, 1994), the normalized FxLMS (NFxLMS) can be given as

$$
\boldsymbol{w}(n+1)=\boldsymbol{w}(n)+\mu(n) e(n) \hat{\boldsymbol{x}}_{s}(n),
$$

where normalized time-varying step size parameter $\mu(n)$ is computed as

$$
\mu(n)=\frac{\tilde{\mu}}{\left\|\hat{\boldsymbol{x}}_{s}(n)\right\|_{2}^{2}+\delta}
$$

where $\tilde{\mu}$ is fixed step size parameter, $\left\|\hat{\boldsymbol{x}}_{s}(n)\right\|_{2}$ is $l_{2}$-norm of the filtered-reference signal vector that can be computed from current available data, and $\delta$ is small positive number added to avoid division by zero. When the reference signal has a large peak, its energy would increase, and this would in turn decrease the effective step size of NFxLMS algorithm. As stated earlier, the error signal is also peaky in nature and its effect must also be taken into account. We 
propose following modified normalized step size for FxLMS algorithm of Eq. (27)

$$
\mu(n)=\frac{\tilde{\mu}}{\left\|\hat{\boldsymbol{x}}_{s}(n)\right\|_{2}^{2}+E_{e}(n)+\delta}
$$

where $E_{e}(n)$ is energy of the residual error signal $e(n)$ that can be estimated online using a lowpass estimator as

$$
E_{e}(n)=\lambda E_{e}(n-1)+(1-\lambda) e^{2}(n),
$$

where $\lambda$ is the forgetting factor $(0.9<\lambda<1)$. It is worth mentioning that the proposed modified normalized FxLMS (MNFxLMS) algorithm, comprising Eqs. (27), (29) and (30), does not require estimation of thresholding parameters $\left[c_{1}, c_{2}\right]$.

\subsection{FxLMP Algorithm and proposed modifications}

For stable distributions, the moments only exist for the order less than the characteristic exponent (Shao \& Nikias, 1993), and hence the MSE criterion which is bases for FxLMS algorithm, is not an adequate optimization criterion. In (Leahy et al., 1995), the filtered-x least mean $p$-power (FxLMP) algorithm has been proposed, which is based on minimizing a fractional lower order moment ( $p$-power of error) that does exist for stable distributions. For some $0<p<\alpha$, minimizing the $p$ th moment $J(n)=E\left\{|e(n)|^{p}\right\} \approx|e(n)|^{p}$, the stochastic gradient method to update $W(z)$ is given as (Leahy et al., 1995)

$$
\boldsymbol{w}(n+1)=\boldsymbol{w}(n)+\mu_{w} p(e(n))^{<p-1>} \hat{\boldsymbol{x}}_{s}(n),
$$

where the operation $(z)^{<a>}$ is defined as

$$
(z)^{<a>} \equiv|z|^{a} \operatorname{sgn}(z),
$$

where $\operatorname{sgn}(z)$ is sign function being defined as

$$
\operatorname{sgn}(z)=\left\{\begin{array}{r}
1, z>0 \\
0, z=0 \\
-1, z<0
\end{array}\right.
$$

It has been shown that FxLMP algorithm with $p<\alpha$ shows good robustness to ANC of impulsive noise. Our objective in this contribution is to improve the convergence performance of the FxLMP algorithm proposed in (Leahy et al., 1995). Based on our extensive simulation studies, we propose two modified versions of the FxLMP algorithm.

The first proposed algorithm attempts to improve the robustness of FxLMP algorithm by using the modified reference and error signals as given in Eqs. (22) and (23), respectively. Thus considering the FxLMP algorithm (Leahy et al., 1995) given in Eq. (31), a modified FxLMP (MFxLMP) algorithm for ANC of impulse noise is given as ${ }^{2}$

$$
\mathbf{w}(n+1)=\mathbf{w}(n)+\mu_{w} p\left(e^{\prime \prime}(n)\right)^{<p-1>} \boldsymbol{x}_{s}^{\prime \prime}(n) .
$$

As done with the FxLMS algorithm, the second modification is based on normalizing the step size parameter and hence, it avoids selection of the thresholding parameters $\left[c_{1}, c_{2}\right]$. In

\footnotetext{
${ }^{2}$ Some preliminary results regarding this algorithm were presented at IEEE ICASSP 2009 (Akhtar \& Mituhahsi, 2009b).
} 


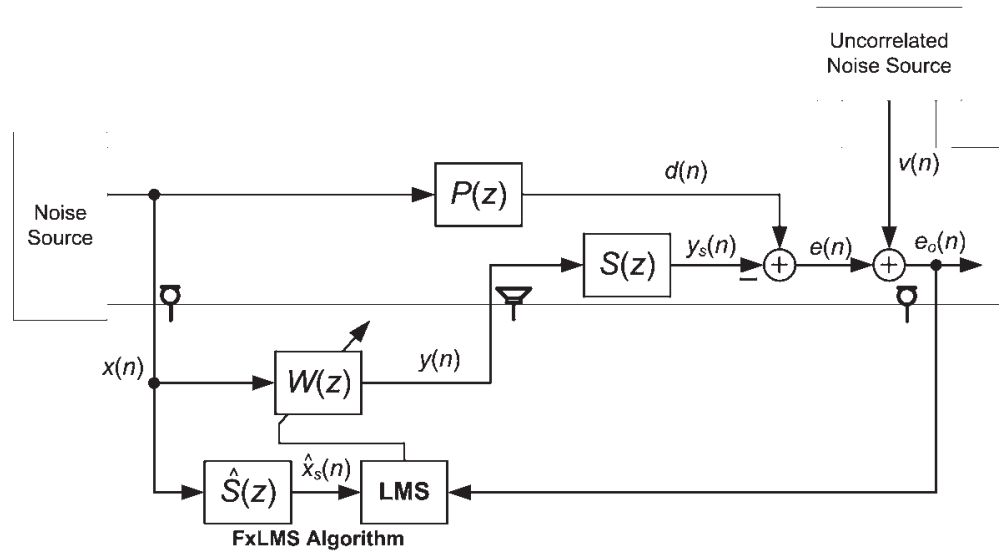

Fig. 4. Block diagram of FxLMS algorithm based single-channel feedforward ANC systems in the presence of uncorrelated disturbance $v(n)$ at the error microphone.

(Aydin et al., 1999), the concept of NLMS algorithm (Douglas, 1994) has been extended to LMP algorithm and a normalized LMP (NLMP) algorithm has been proposed where step size is normalized by the energy of reference signal vector. By extending this idea to FxLMP algorithm (Leahy et al., 1995), the normalized FxLMP (NFxLMP) can be given as

$$
\boldsymbol{w}(n+1)=\boldsymbol{w}(n)+\mu(n) p(e(n))^{<p-1>} \hat{\boldsymbol{x}}_{\boldsymbol{s}}(n),
$$

where normalized time-varying step size parameter $\mu(n)$ is computed as

$$
\mu(n)=\frac{\tilde{\mu}}{\left\|\hat{\boldsymbol{x}}_{s}(n)\right\|_{p}^{p}+\delta}
$$

where $\left\|\hat{\boldsymbol{x}}_{s}(n)\right\|_{p}$ is $p$ th norm computed from current filtered-reference signal vector. Since the error signal $e(n)$ is also peaky in nature and its effect must also be taken into account, we propose following modified normalized step size for FxLMP algorithm of Eq. (35):

$$
\mu(n)=\frac{\tilde{\mu}}{\left\|\hat{\boldsymbol{x}}_{s}(n)\right\|_{p}^{p}+E_{e}(n)+\delta},
$$

where $E_{e}(n)$ is energy of the residual error signal $e(n)$. Thus a modified normalized FxLMP (MNFxLMP) algorithm is suggested comprising Eqs. (35), (37) and (30).

In this section we have suggested ad hoc modifications to the existing adaptive algorithms for ANC of impulsive noise. The simulation results presented later in Section 5.1 demonstrate that these modifications greatly improve robustness of ANC system for the impulsive noise sources.

\section{Mitigating uncorrelated disturbance}

The FxLMS algorithm is widely used in ANC systems, however performance of the FxLMS algorithm in steady state will be degraded due to presence of an uncorrelated disturbance 
at the error microphone, shown as $v(n)$ in Fig. 4. This situation arises in many real-world applications. For example, in electronic mufflers for automobiles (Kuo \& Gan, 2004), the undesired disturbance such as the noises generated by other passing-by automobiles will affect the stability and performance of the ANC systems. In industrial installations, neighboring machinery near to the location of error microphone may generate uncorrelated disturbance. In the presence of uncorrelated disturbance, $v(n)$, the error signal picked-up by the error microphone is given as

$$
e_{o}(n)=e(n)+v(n)=\left[d(n)-y_{s}(n)\right]+v(n),
$$

and hence, the update equation for FxLMS algorithm for $W(z)$ can be written as

$$
\boldsymbol{w}(n+1)=\boldsymbol{w}(n)+\mu_{w} e(n) \hat{\boldsymbol{x}}_{s}(n)+\mu_{w} v(n) \hat{\boldsymbol{x}}_{s}(n) .
$$

It is evident that the adaptation is perturbed by the uncorrelated noise component $v(n)$, and as shown in (Sun \& Kuo, 2007), the steady-state performance of the FxLMS algorithm will be degraded significantly. Furthermore, $v(n)$ appearing uncontrolled at the error microphone degrades the noise reduction performance of the ANC system.

Up to the best knowledge of Authors, a little research has been done to cope with the uncorrelated disturbance problem. In (Kuo \& Ji, 1996), an adaptive algorithm consisting of two interconnected adaptive notch filters is proposed to reduce the disturbance problem. However, this algorithm is effective only for narrowband, single-frequency ANC systems. In (Sun \& Kuo, 2007), this algorithm has been generalized to multifrequency narrowband feedforward ANC systems using a single high-order adaptive filter, and a cascaded ANC system is proposed. This method improves the convergence of the FxLMS algorithm, however, cannot mitigate the effect of the uncorrelated disturbance $v(n)$ from the residual noise $e(n)$. One solution to this problem of uncorrelated disturbance is offered by a hybrid ANC comprising feedforward and feedback control strategies (Esmailzadeh et al., 2002). The feedforward ANC attenuates the primary noise that is correlated with the reference signal, whereas the feedback ANC takes care of the narrowband components of noise that are not observed by the reference sensor. We observe that the performance of the hybrid ANC system degrades in certain situations, as explained later in this section.

\subsection{Existing solutions for uncorrelated disturbance}

The main idea of cascading ANC system (Sun \& Kuo, 2007) is to update the adaptive filter $W(z)$ using estimate of the desired error signal $e(n)$ instead of using a disturbed error signal $e_{0}(n)$. The block diagram of cascading ANC system is shown in Fig. 5, where the adaptive filter $H(z)$ is introduced to estimate the desired error signal $e(n)$.

It is evident that $H(z)$ is excited by the reference signal $x(n)$, and the error signal $e_{o}(n)$ is used as a desired response for its adaptation. Thus output of $H(z), y_{h}(n)$, converges to that part in $e_{0}(n)$ which is correlated with $x(n)$. From Eqs. (1), (3), and (4), it is clear that in $e_{0}(n)$ given in Eq. (38), $e(n)=\left[d(n)-y_{s}(n)\right]$ is correlated with $x(n)$ and $v(n)$ is the uncorrelated part. Hence, when $H(z)$ converges, its output converges to $y_{h}(n) \approx e(n)=\left[d(n)-y_{s}(n)\right]$, which is the desired error signal for the adaptation of $W(z)$. Thus FxLMS algorithm for this cascading $\mathrm{ANC}$ is given as

$$
\boldsymbol{w}(n+1)=\boldsymbol{w}(n)+\mu_{w} y_{h}(n) \hat{\boldsymbol{x}}_{s}(n) .
$$




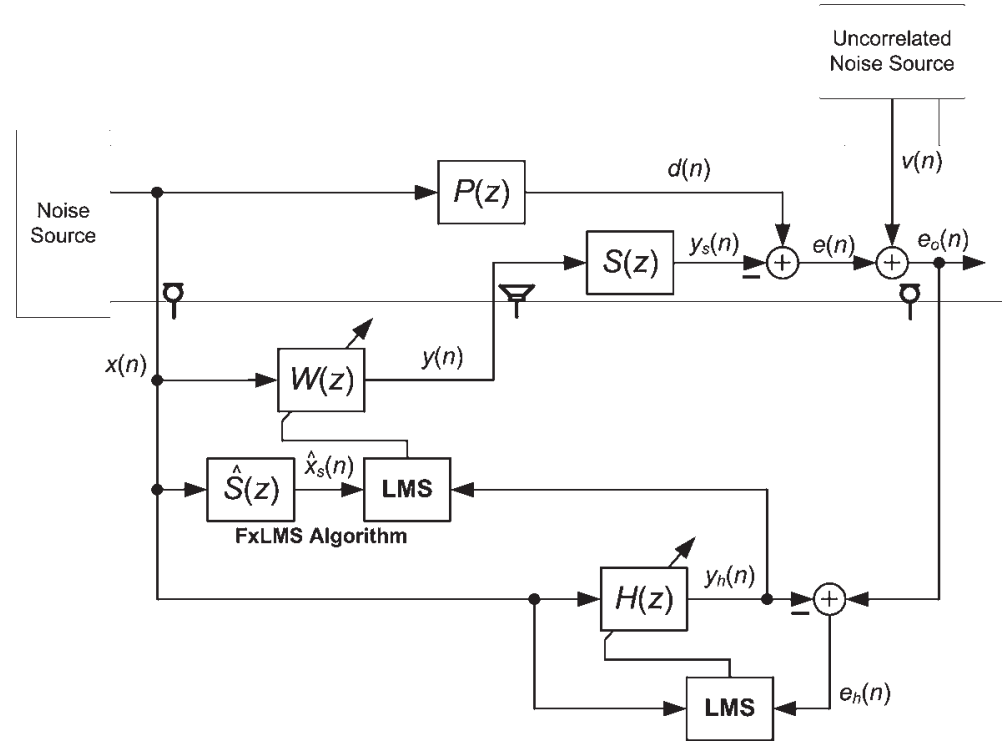

Fig. 5. Block diagram of the cascading ANC system for improving adaptation of FxLMS algorithm in the presence of uncorrelated disturbance $v(n)$ (Sun \& Kuo, 2007).

Since a disturbance free error signal is used, cascading ANC improves the convergence of the FxLMS algorithm. However, it cannot mitigate effect of the uncorrelated disturbance $v(n)$ from the residual noise $e_{0}(n)$.

One solution for ANC of correlated and uncorrelated disturbances would be to consider a hybrid ANC system comprising feedforward ANC $W(z)$ and feedback ANC B( $z)$ as shown in Fig. 6 (Kuo \& Morgan, 1996). We assume that the two noise sources are independent from each other, and thus the primary disturbances $d(n)$ and $v(n)$ are uncorrelated with each other. The reference signal $x(n)$ from the reference microphone is correlated with $d(n)$ and is input to feedforward ANC $W(z)$. The total cancelling signal is sum of outputs of $W(z)$ and $B(z)$ and is passed through $S(z)$ to generate the residual error signal $e_{0}(n)$ as

$$
e_{0}(n)=\left[d(n)-y_{s}(n)\right]+\left[v(n)-g_{s}(n)\right] .
$$

This error signal is used in the FxLMS algorithm for both $W(z)$ and $B(z)$. Comparing $e_{0}(n)$ in Eq. (41) with $e(n)$ in Eq. (4) and with $e_{b}(n)$ in Eq. (8), we see that $e_{o}(n)$ comprises two components. The first component is required for the adaptation of feedforward ANC $W(z)$ and acts as a disturbance for feedback ANC $B(z)$. The second component plays exactly the reverse role, i.e., a disturbance for $W(z)$ and desired error signal for adaptation of $B(z)$. The reference signal for $W(z), x(n)$, is given by the reference microphone, and the reference signal for $B(z), u(n)$, is internally generated as

$$
\begin{aligned}
u(n) & =e_{o}(n)+\hat{y}_{s}(n)+\hat{g}_{s}(n) \\
& =\left[d(n)-y_{s}(n)+\hat{y}_{s}(n)\right]+\left[v(n)-g_{s}(n)+\hat{g}_{s}(n)\right] \\
& \approx \hat{d}(n)+\hat{v}(n) .
\end{aligned}
$$




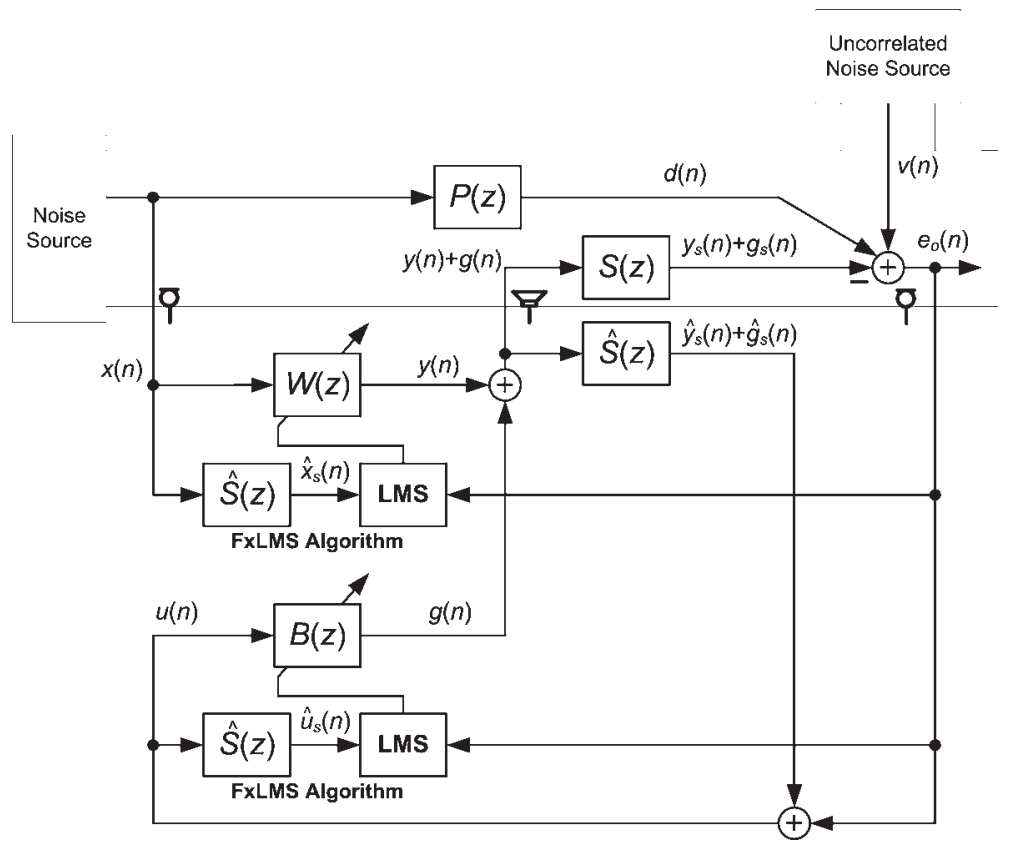

Fig. 6. Block diagram of conventional hybrid ANC system with combination of feedforward ANC $W(z)$ and feedback ANC $B(z)$.

Thus the reference signal $u(n)$ comprises two parts; estimates of disturbances $d(n)$ and $v(n)$. Since objective of the feedback ANC $B(z)$ is to cancel only uncorrelated primary noise $v(n)$, the presence of $\hat{d}(n)$ (which may be broadband and unpredictable in general) gives a corrupted reference signal for $B(z)$. Thus, both $W(z)$ and $B(z)$ are adapted using inappropriate error signals and may converge slowly. Furthermore, $B(z)$ is excited by a corrupted reference signal and might not converge at all, making whole ANC system unstable. From above discussion, we conclude that

- the cascading ANC (Sun \& Kuo, 2007) improves the convergence of the FxLMS algorithm, however, it cannot remove the effect of the uncorrelated disturbance from the residual noise, and that

- the conventional hybrid ANC (Kuo \& Morgan, 1996) can provide control over correlated and uncorrelated noise sources, however, its performance might be poor, as ANC filters are using inappropriate error and/or reference signals.

In order to solve these limitations of the existing methods, a modified hybrid ANC is developed as explained in the next section.

\subsection{Modified hybrid ANC System}

The block diagram of modified hybrid ANC system is shown in Fig. 7 (Akhtar \& Mituhahsi, 2011), and as shown, this method comprises three adaptive filters: 1) a feedforward ANC filter $W(z)$ to cancel the primary noise $d(n), 2)$ a feedback ANC filter $B(z)$ to cancel the uncorrelated disturbance $v(n)$, and 3 ) a supporting filter $H(z)$. The $W(z)$ is excited by the reference signal 


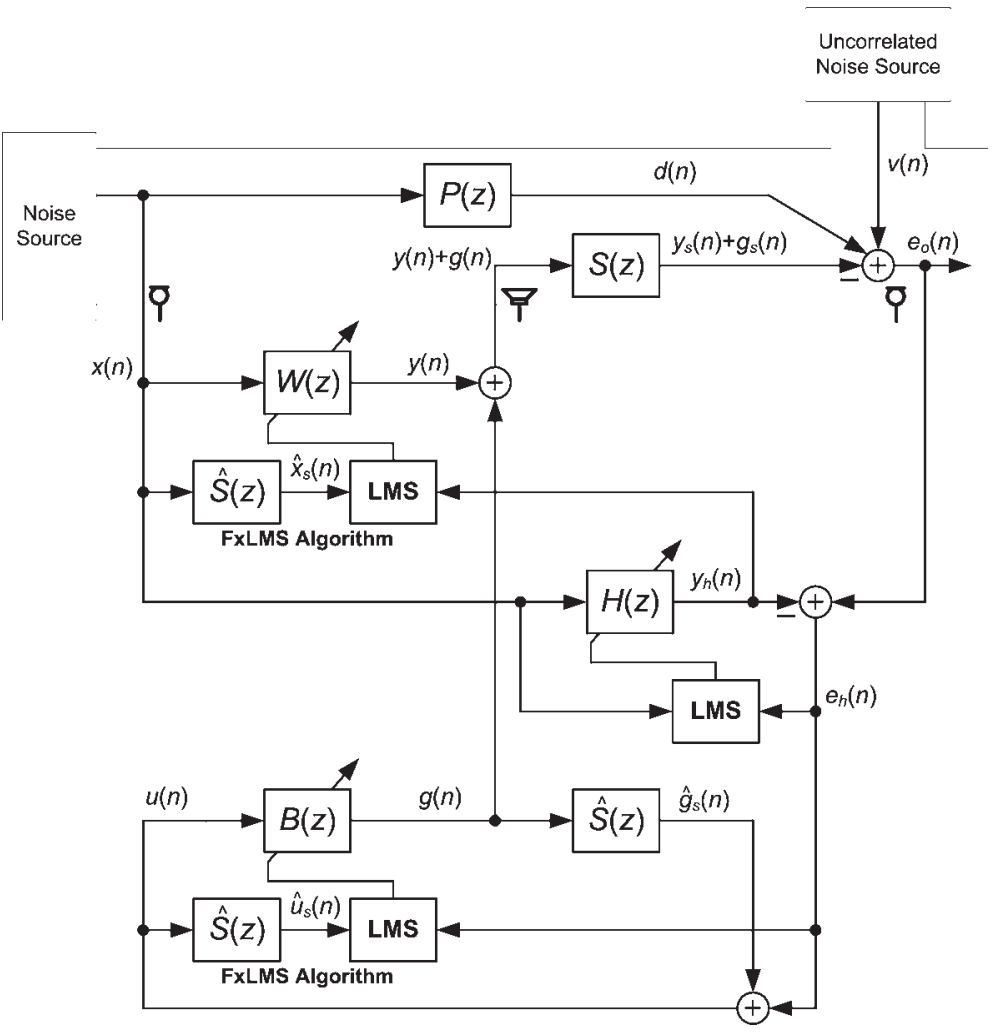

Fig. 7. Block diagram of a modified hybrid ANC system for controlling correlated and uncorrelated noise sources.

$x(n)$, and the $B(z)$ is excited by an internally generated reference signal $u(n)$. Both ANC filters $W(z)$ and $B(z)$ are adapted by FxLMS algorithms.

The residual error signal $e_{0}(n)$ is given in Eq. (41) and as explained earlier, the first term is desired error signal for the adaptation of $W(z)$ and second term is desired error signal for $B(z)$. To achieve cancellation [ideally $e_{0}(n)=0$ ] $W(z)$ needs to be excited by the input correlated with $d(n)$ [the reference signal $x(n)$ is indeed that input], and $B(z)$ needs to be excited by the input correlated with $v(n)$ [such input is not available directly and needs to be generated internally].

As shown in Fig. 7, the third adaptive filter $H(z)$ is excited by the reference signal $x(n)$, and its output, $y_{h}(n)$, is given as

$$
y_{h}(n)=\boldsymbol{h}^{T}(n) \boldsymbol{x}(n),
$$

where $\boldsymbol{h}(n)$ is the tap-weight vector for $H(z)$. The residual error signal $e_{0}(n)$ given in Eq. (41), is used as a desired response, and the error signal for LMS equation of $H(z), e_{h}(n)$, is 
computed as

$$
\begin{aligned}
e_{h}(n) & =e_{o}(n)-y_{h}(n) \\
& =\left[d(n)-y_{s}(n)\right]+\left[v(n)-g_{s}(n)\right]-y_{h}(n),
\end{aligned}
$$

and $H(z)$ is adapted using LMS algorithm as

$$
\boldsymbol{h}(n+1)=\boldsymbol{h}(n)+\mu_{h} e_{h}(n) \boldsymbol{x}(n),
$$

where $\mu_{h}$ is the step size for $H(z)$. Since $H(z)$ is excited by $x(n)$, minimizing the error signal $e_{h}(n)$ means that the output of $H(z), y_{h}(n)$, would converge to that part in Eq. (44) which is correlated with $x(n)$, thus

$$
y_{h}(n) \rightarrow\left[d(n)-y_{s}(n)\right],
$$

and hence $e_{h}(n)$ would converge to the remaining uncorrelated part

$$
e_{h}(n) \rightarrow\left[v(n)-g_{s}(n)\right] .
$$

Comparing Eq. (46) with Eq. (4), we see that that $y_{h}(n)$ can be used as an error signal for adaptation of $W(z)$, and hence FxLMS algorithm for $W(z)$ is given as

$$
\boldsymbol{w}(n+1)=\boldsymbol{w}(n)+\mu_{w} y_{h}(n) \hat{\boldsymbol{x}}_{\boldsymbol{s}}(n)
$$

and similarly, comparing Eq. (47) with Eq. (8), we observe that $e_{h}(n)$ can be used as an error signal for feedback ANC filter $B(z)$, and corresponding FxLMS algorithm for $B(z)$ is thus given as

$$
\boldsymbol{b}(n+1)=\boldsymbol{b}(n)+\mu_{b} e_{h}(n) \hat{\boldsymbol{u}}_{s}(n)
$$

where $\hat{\boldsymbol{u}}_{s}(n)=\hat{\boldsymbol{s}}(n) * \boldsymbol{u}(n)$, and the reference signal $\boldsymbol{u}(n)$ is generated as

$$
\begin{aligned}
u(n) & =e_{h}(n)+\hat{g}_{s}(n) \\
& \approx\left[v(n)-g_{s}(n)\right]+\hat{g}_{s}(n) \\
& \rightarrow \hat{v}(n) .
\end{aligned}
$$

Comparing Eq. (50) with Eq. (42), we see that the input $u(n)$ for feedback ANC filter $B(z)$ in the modified hybrid ANC of Fig. 7 is equal to estimate of only uncorrelated noise source $v(n)$. A comparison between the modified hybrid ANC and existing approaches is as given below:

- The modified hybrid ANC provides control over both correlated and uncorrelated disturbances, where as cascading ANC of (Sun \& Kuo, 2007) can only improve the convergence of $W(z)$, but cannot reduce the uncorrelated disturbance.

- In modified hybrid ANC, role of $H(z)$ is partly same as that in Sun's method. It generates desired error signal for adaptation of $W(z)$ to provide cancellation for correlated disturbance signal $d(n)$. Furthermore, it is used to generate appropriate signals for adaptation of $B(z)$ to cancel uncorrelated disturbance $v(n)$. Therefore, as compared with the conventional hybrid ANC, proposed structure results in generating appropriate signals for two adaptive filter, and hence, an improved convergence and residual noise reduction is expected.

- The modified hybrid ANC comprises three adaptive filters and its computational complexity is higher than the existing approaches. This increased computational 


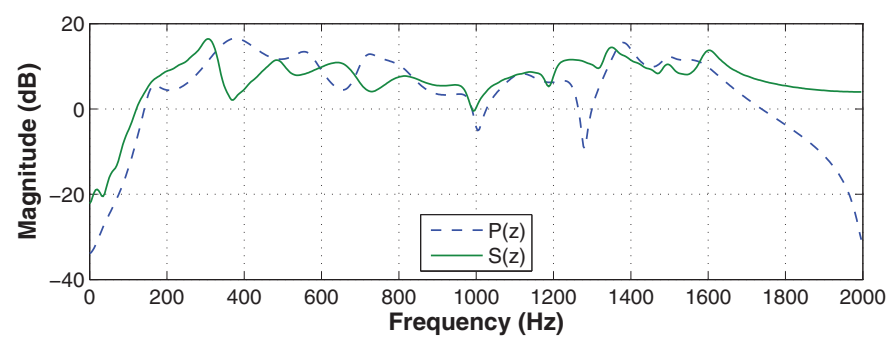

(a)

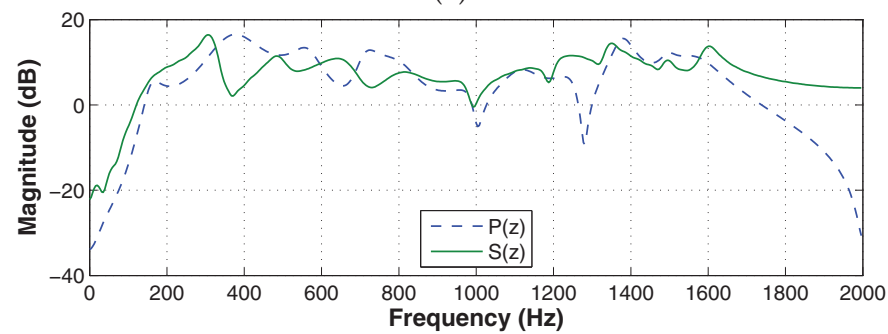

(b)

Fig. 8. Frequency response of the primary path $P(z)$ and secondary path $S(z)$. (a) Magnitude response and $(b)$ phase response.

complexity may be considered as the price paid for improved performance. In fact, the simulation results presented in Section 5.2 demonstrate that modified hybrid ANC achieves the performance which is not possible with neither cascading ANC nor conventional hybrid ANC working alone.

\section{Computer simulations}

In this section results of computer simulation are presented for two case studies discussed in this chapter, viz., ANC for impulsive noise sources, and mitigating uncorrelated disturbance. The acoustic paths are modeled using data provided in the disk attached with (Kuo \& Morgan, 1996). Using this data $P(z)$ and $S(z)$ are modeled as FIR filter of length 256 and 128 respectively. The characteristics of the acoustic paths are shown in Fig. 8. It is assumed that the secondary path modeling filter $\hat{S}(z)$ is exactly identified as $S(z)$.

\subsection{ANC for impulsive noise sources}

The simulation setup is same as shown in Fig. 1, where noise source is assumed to be impulsive and the ANC filter $W(z)$ is selected as an FIR filter of tap-weight length $L_{w}=192$. The performance comparison is done on the basis of mean noise reduction (MNR), being defined as

$$
\operatorname{MNR}(n)=E\left\{\frac{A_{e}(n)}{A_{d}(n)}\right\},
$$

where $E\{\cdot\}$ denotes expectation or ensemble averaging of quantity inside, and $A_{e}(n)$ and $A_{d}(n)$ are estimates of absolute values of residual error signal $e(n)$ and disturbance signal 


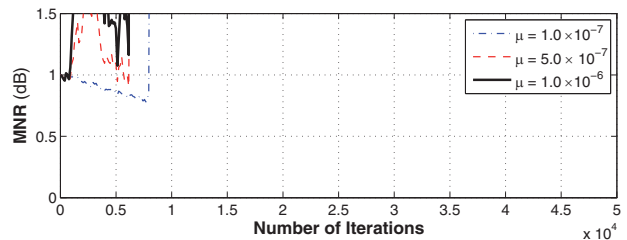

(a)

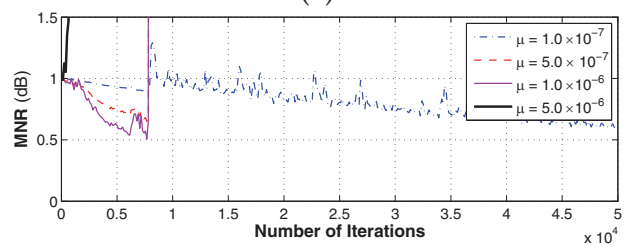

(c)

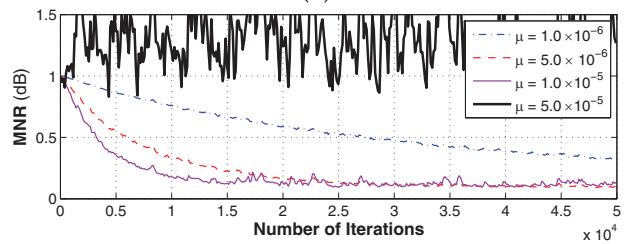

(e)

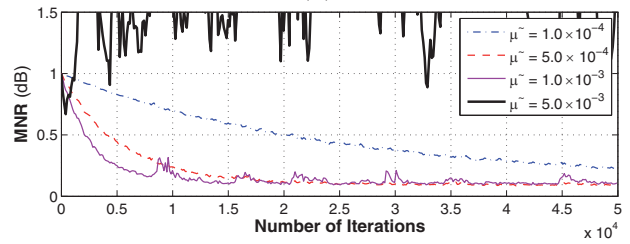

$(\mathrm{g})$

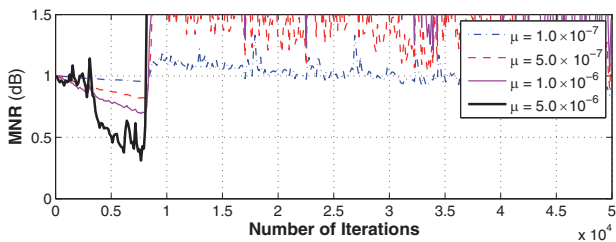

(b)

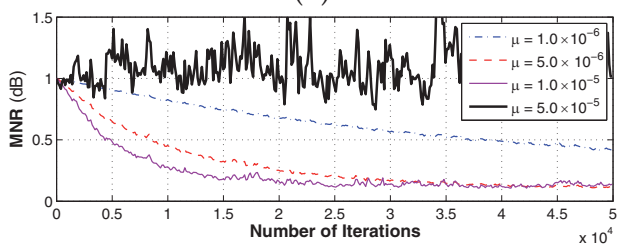

(d)

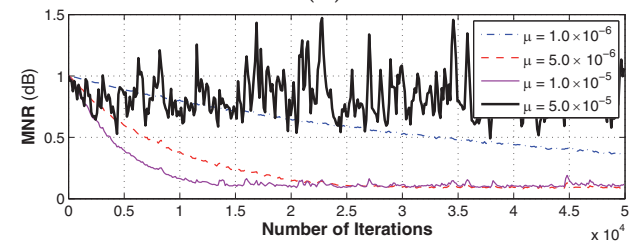

(f)

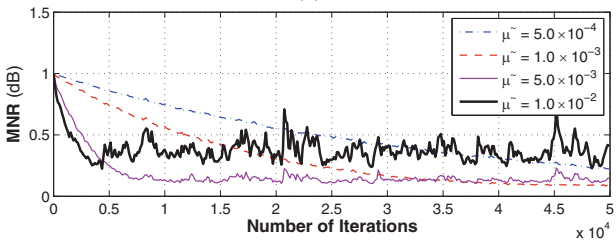

(h)

Fig. 9. Mean noise reduction (MNR) curves for various algorithms for ANC of impulsive noise with $\alpha=1.45$. (a) FxLMS algorithm, (b) FxLMP algorithm, (c) Sun's algorithm, (d) Modified-Sun's algorithm, (e) MFxLMS algorithm, (f) MFxLMP algorithm, (g) MNFxLMS algorithm, and (h) MNFxLMP algorithm.

$d(n)$, respectively, at the location of error microphone. These estimates are obtained using lowpass estimators as

$$
A_{r}(n)=\lambda A_{r}(n-1)+(1-\lambda)|r(n)|
$$

where $|\cdot|$ is the absolute value of quantity, and $\lambda$ is same as defined in Eq. (30).

The reference noise signal $x(n)$ is modeled by standard $S \alpha S$ process with $\alpha=1.45$ (which corresponds to a very peaky distribution more toward Cauchy distribution), and $\alpha=$ 1.65 (corresponding to distribution towards Gaussian distribution). All simulation results presented below are averaged over 25 realization of the process. Extensive simulations are carried out to find appropriate values for the thresholding parameters $\left[c_{1}, c_{2}\right]$, and are selected as: $[0.01,99.99]$ for Sun's algorithm in Eq. (17), [0.5, 99.5] for modified-Sun's algorithm in Eq. (21), and [1,99] for MFxLMS algorithm in Eq. (24). The detailed simulation results 


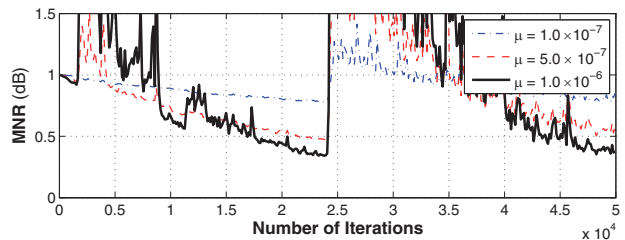

(a)

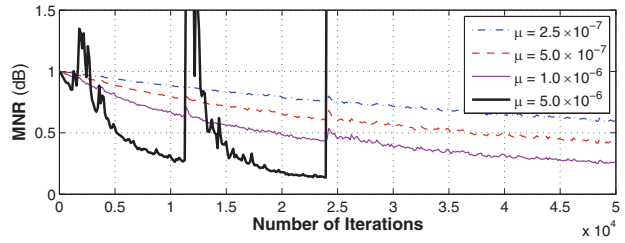

(c)

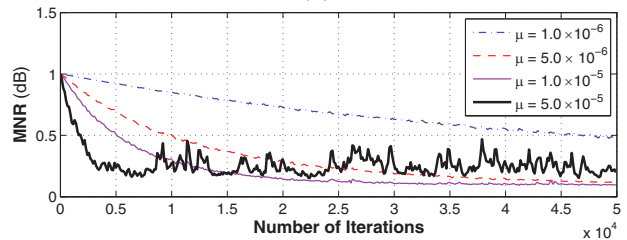

(e)

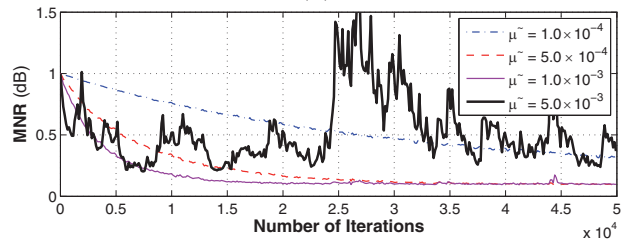

$(\mathrm{g})$

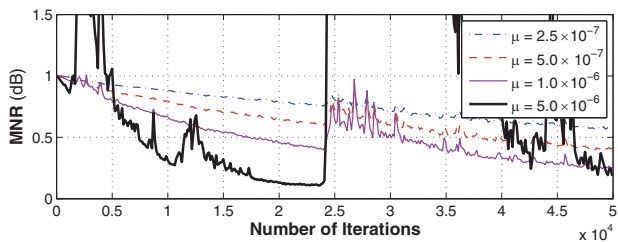

(b)

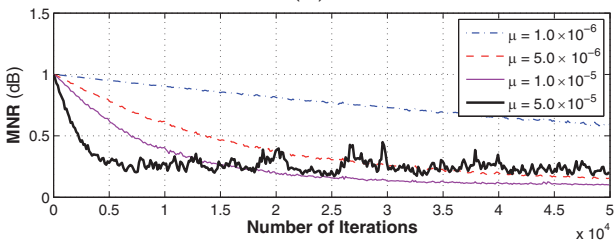

(d)

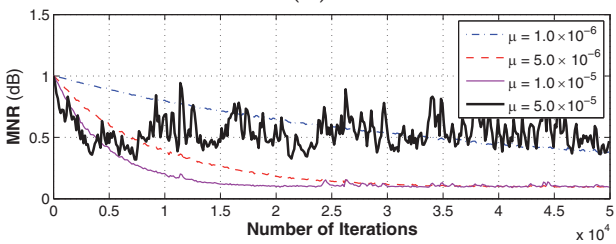

(f)

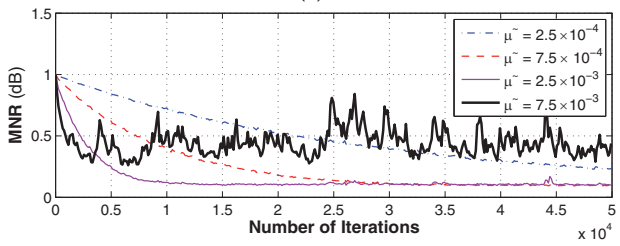

(h)

Fig. 10. Mean noise reduction (MNR) curves for various algorithms for ANC of impulsive noise with $\alpha=1.65$. (a) FxLMS algorithm, (b) FxLMP algorithm, (c) Sun's algorithm, (d) Modified-Sun's algorithm, (e) MFxLMS algorithm, (f) MFxLMP algorithm, (g) MNFxLMS algorithm, and (h) MNFxLMP algorithm.

for two cases are given in Figs. 9 and 10, respectively, where the objective is to study the effect of step size parameter. It is seen that, the FxLMS algorithm is not able to provide ANC for impulsive noise, even for a very small step size. Furthermore, in comparison with the Authors' algorithms, the performance of Sun's algorithm and FxLMP algorithm is very poor. On the basis of best results for the respective algorithms, the performance comparison for two cases is shown in Figs. 11 and 12, respectively. These results show that the proposed algorithms outperform the existing algorithms and, among the algorithms discussed in Section 3, appears as a best choice for ANC of $S \alpha S$ impulsive noise. 


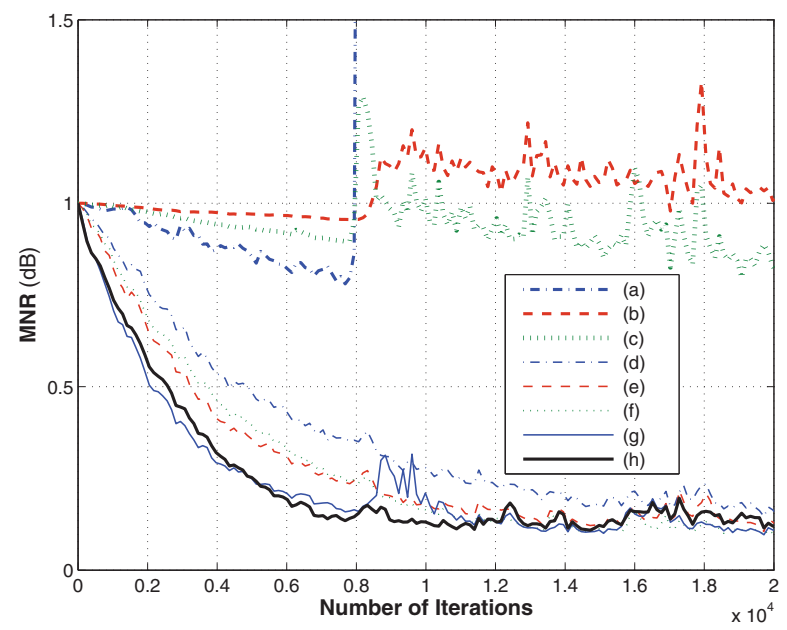

Fig. 11. Performance comparison between various algorithms for ANC of impulsive noise with $\alpha=1.45$. (a) FxLMS algorithm $\left(\mu=1 \times 10^{-7}\right)$, (b) FxLMP algorithm $\left(\mu=1 \times 10^{-7}\right)$, (c) Sun's algorithm $\left(\mu=1 \times 10^{-7}\right)$, (d) Modified-Sun's algorithm $\left(\mu=1 \times 10^{-5}\right)$, (e) MFxLMS algorithm $\left(\mu=1 \times 10^{-5}\right.$, (f) MFxLMP algorithm $\left(\mu=1 \times 10^{-5}\right.$, (g) MNFxLMS algorithm $\left(\tilde{\mu}=1 \times 10^{-3}\right)$, and (h) MNFxLMP algorithm $\left(\tilde{\mu}=5 \times 10^{-3}\right)$.

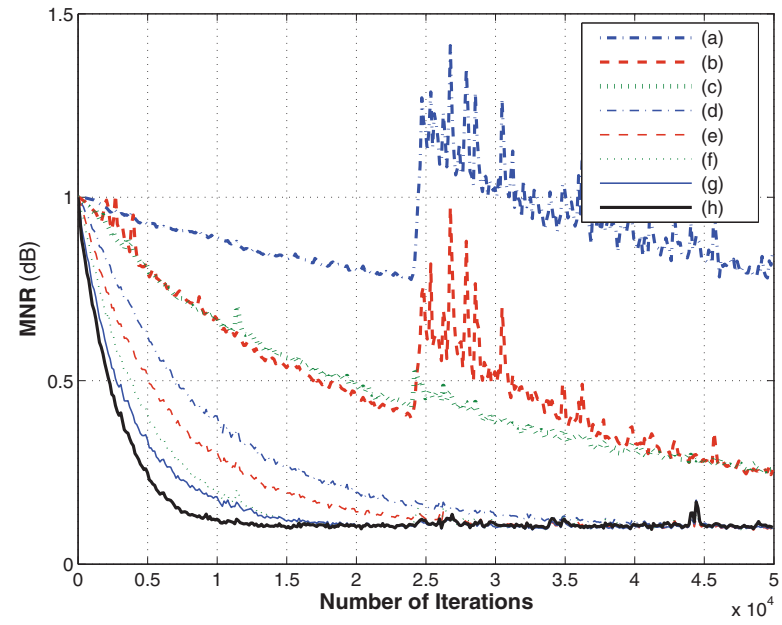

Fig. 12. Performance comparison between various algorithms for ANC of impulsive noise with $\alpha=1.65$. (a) FxLMS algorithm $\left(\mu=1 \times 10^{-7}\right)$, (b) FxLMP algorithm $\left(\mu=1 \times 10^{-6}\right)$, (c) Sun's algorithm $\left(\mu=1 \times 10^{-6}\right)$, (d) Modified-Sun's algorithm $\left(\mu=1 \times 10^{-5}\right)$, (e) MFxLMS algorithm $\left(\mu=1 \times 10^{-5}\right.$, (f) MFxLMP algorithm $\left(\mu=1 \times 10^{-5}\right.$, (g) MNFxLMS algorithm $\left(\tilde{\mu}=1 \times 10^{-3}\right)$, and (h) MNFxLMP algorithm $\left(\tilde{\mu}=2.5 \times 10^{-3}\right)$. 


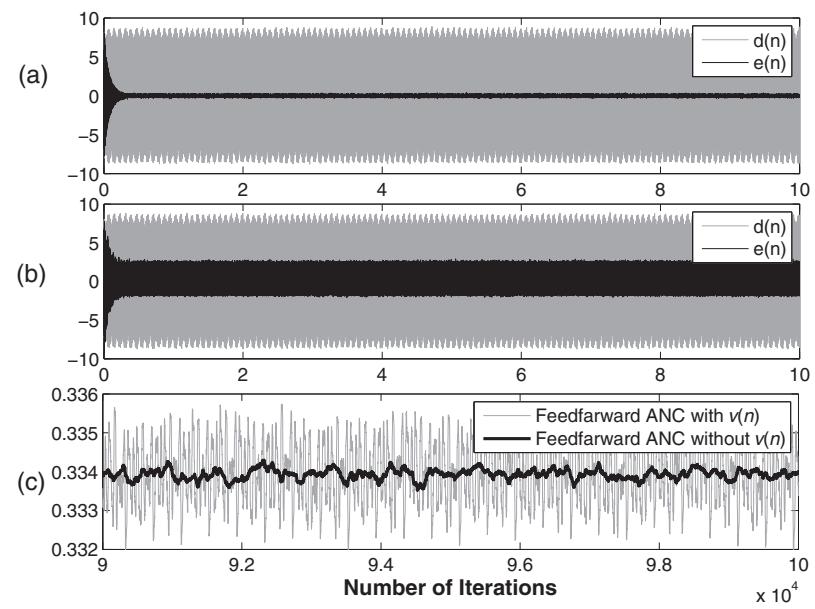

Fig. 13. Simulation results for mitigating uncorrelated disturbance $v(n)$. (a) Feedforward ANC without uncorrelated disturbance $v(n)$. (b) Feedforward ANC in the presence of uncorrelated disturbance $v(n)$. (c) Norm of weight vector, $\|\boldsymbol{w}(n)\|$, at steady state.

\subsection{Mitigating uncorrelated disturbance}

In this section we present simulation results for various methods studied for mitigating uncorrelated disturbances. Essentially, we consider following methods in our study:

1. feedforward ANC shown in Fig. 4,

2. cascading ANC system of Fig. 5,

3. conventional hybrid ANC of Fig. 6, and

4. modified hybrid ANC of Fig. 7.

The noise source $x(n)$ is assumed as a unit variance narrowband signal composed of three sinusoids with frequencies of 165,290 , and $410 \mathrm{~Hz}$. A white noise with variance 0.001 is added to count for measurement noise at the reference microphone. The uncorrelated disturbance $v(n)$ is another unit variance narrowband signal comprising three sinusoids with frequencies of 250,350 , and $500 \mathrm{~Hz}$, and a white noise with variance 0.001 is added to count for measurement noise at the error microphone. The sampling frequency is $4 \mathrm{kHz}$, and the results shown are average of 10 realizations. The adaptive filters $W(z), B(z)$, and $H(z)$ are selected as FIR filters of tap-weight lengths 192, 192, and 32, respectively. All adaptive filters are initialized by null vectors of an appropriate order. The step sizes are selected experimentally, such that fast and stable performance is obtained and are adjusted as, feedforward ANC: $\mu_{w}=1 \times 10^{-5}$, cascading ANC: $\mu_{w}=1 \times 10^{-5}, \mu_{h}=1 \times 10^{-3}$, conventional hybrid ANC: $\mu_{w}=1 \times 10^{-5}, \mu_{b}=1 \times 10^{-6}$, and modified hybrid ANC: $\mu_{w}=1 \times 10^{-5}, \mu_{b}=1 \times 10^{-5}$, $\mu_{h}=5 \times 10^{-3}$.

The effect of the uncorrelated disturbance $v(n)$, on the performance of feedforward ANC of Fig. 4, is studied in Fig. 13. We see that feedforward ANC cannot control the uncorrelated disturbance $v(n)$ appearing at the error microphone, and hence, its noise reduction performance is degraded, as indicated by increased level of residuals noise $e(n)$ (Fig. 13 (a) and (b)). Furthermore, the uncorrelated disturbance disturbs the convergence of ANC 


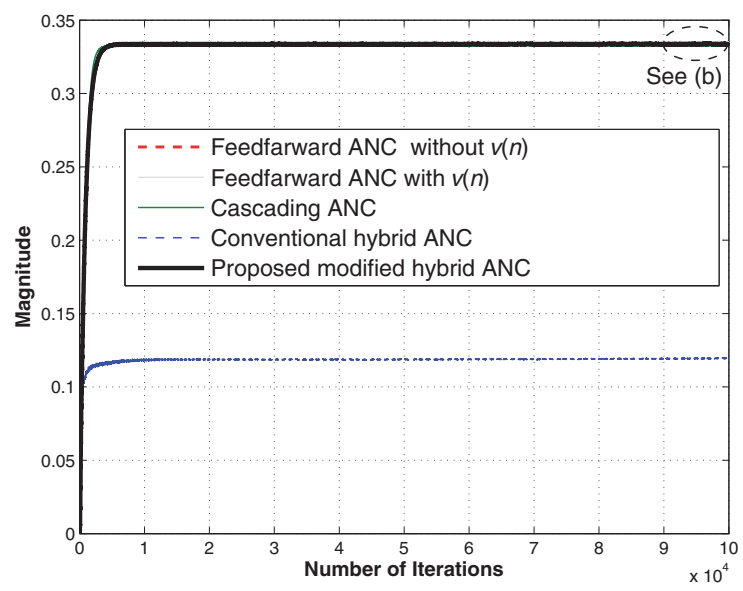

(a)

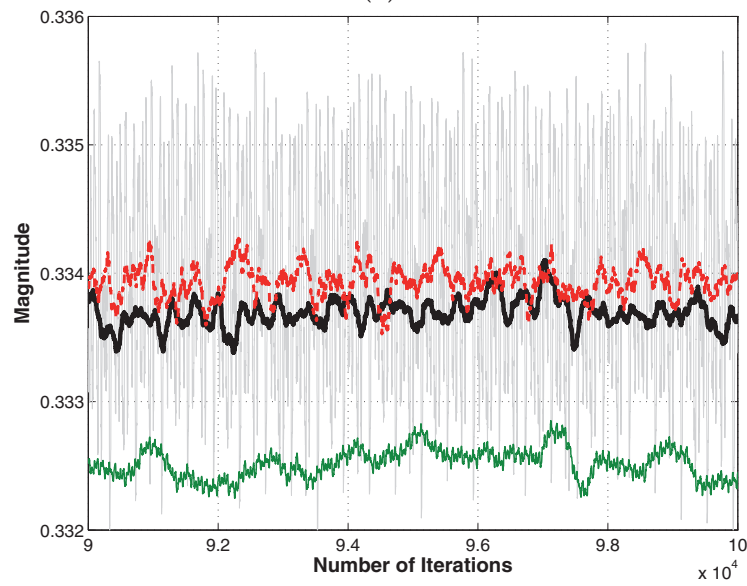

(b)

Fig. 14. Simulation results for mitigating uncorrelated disturbance $v(n)$. (a) Convergence of norm of tap-weights for ANC filter $W(z),\|\boldsymbol{w}(n)\|$. (b) Zoomed curves for region marked by dashed ellipse in (a).

filter, as shown in Fig. 13 (c). Our objective is to improve the noise reduction performance and have stable steady state convergence of tap-weights.

The adaptation of adaptive filter $W(z)$, in terms of $\|\boldsymbol{w}(n)\|$, which is Euclidean norm of weight vector $\boldsymbol{w}(n)$, is shown in Fig. 14(a). An enlarged view of curves in steady state is shown in Fig. 14(b). We see that the modified hybrid ANC can provide control over the uncorrelated noise source $v(n)$, and hence can remove its effect from the convergence of $W(z)$. In fact, the steady state solution achieved by the modified hybrid ANC is close to that obtained by feedforward ANC without uncorrelated disturbance. We see that, this is a far better performance as compared with the conventional hybrid ANC. 


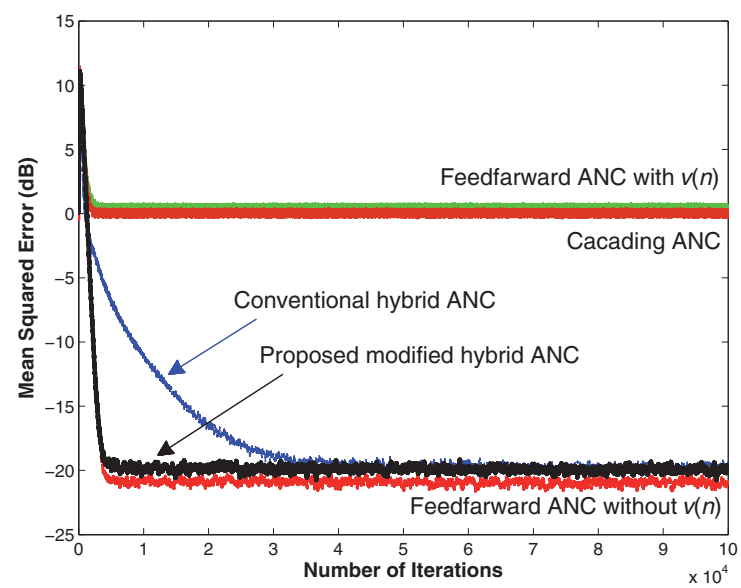

(a)

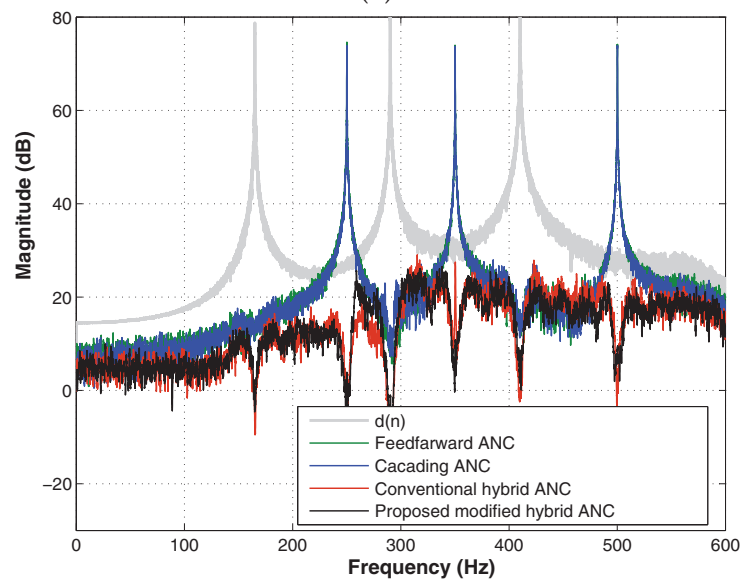

(b)

Fig. 15. Simulation results for mitigating uncorrelated disturbance $v(n)$. (a) Curves for mean squared error (MSE) for various methods. (b) Magnitude spectrum of residual error signal $e(n)$ at steady-state for various methods in comparison with the magnitude spectrum of primary disturbance $d(n)$.

The noise reduction performance, in terms of MSE, for various methods is shown in Fig. 15(a). We see that the uncorrelated disturbance $v(n)$ appearing at the error microphone degrades the noise reduction performance of the feedforward ANC system. The conventional and modified hybrid ANC systems, incorporating a separate ANC filter $B(z)$ for uncorrelated disturbance, give significantly improved noise reduction performance, with the proposed method converging at a faster rate. Fig. 15(b) shows the power spectral density of the residual error signal $e(n)$ in the steady state. The spectrum of the primary disturbance $d(n)$ is also shown as a reference. We see that, as compared with the feedforward ANC, the conventional and modified hybrid ANCs are more effective in removing the uncorrelated disturbance. 


\section{An outlook on recent ANC applications}

In this section, we give a brief overview of new applications of ANC systems in consumer electronics and medical instruments. The first example is active control of speech signals for privacy-phone handsets (Kondo \& Nakagawa, 2007). The idea is to generate out-of-phase speech to cancel the original speech in space, thus allowing private and quiet voice communication in public areas. Developing efficient algorithms and methods for efficient speech emission control in 3D environment requires further research.

In hospitals, there are a lot of life-saving equipment such as breathing and IV pumps that generate impulse-like noises. For example, infant incubators are used in neonatal intensive care units (NICU) to increase the survival of premature and ill infants. The application of ANC for reducing incubator noise in NICU was reported in (Liu et al., 2008), where a nonlinear filtered-X least mean M-estimate algorithm is developed for reducing impulse-like noise in incubators. In Section 3 we have presented some robust algorithms for ANC of impulsive noise sources, and theoretical performance analysis, real-time experiments, and development of more effective ANC algorithms is open for further research.

Recently very interesting results have been reported concerning head mounted ANC for the noise generated during magnetic resonance imaging (MRI) (Kida et al., 2009). The noise generated during MRI is found to be of a narrowband nature, and work presented in (Kida et al., 2009) considers feedback type ANC. It would be interesting to investigate, whether we can get better performance by employing proposed hybrid ANC system for MRI noise.

In the recent years, traffic noise coming from streets, highways, railways, and airports has been of increasing concern. In such situations the positions of noise sources are time varying, and it is necessary to study and develop dynamic ANC systems for moving noise sources relative to the ANC installation. One challenging, yet a very interesting, application would be to study an efficient ANC system for a quiet car interior even when the window or sunroof is open.

In some applications, it is desirable to retain a low-level residual noise with a desired spectral shape or changed noise signature. Active sound quality control (ASQC), which changes amplitudes of noise components with predetermined values, is a useful and important extension of ANC, see (Kuo \& Ji, 1995) for narrowband ASQC and (Kuo \& Yang, 1996) for broadband ASQC. The broadband ASQC algorithm uses a shaping filter to control the residual noise spectrum, and further research is needed to design an appropriate shaping filter. Recently noise reduction for motorcycle helmets is evaluated and some interesting results using hybrid ANC have been reported in (Castañé-Selga \& Sánchez Peña, 2010). In such applications, ASQC systems must be employed so that noise and horn-sounds from the neighboring vehicles are not completely removed.

\section{Concluding remarks}

In this chapter we have provided a comprehensive review of adaptive filtering algorithms developed for ANC systems. We mentioned several signal processing challenges with open problems for further research and development. Furthermore, we presented efficient algorithms and methods for two challenging problems in ANC systems: 1) active control of impulsive-like noise sources, and 2) controlling uncorrelated disturbance for which a correlated reference signal is not available. Finally an outlook of some challenging applications of ANC is provided. 


\section{Acknowledgments}

This research is carried out with funding from Japan Science and Technology (JST) Agency.

\section{References}

Aydin, G. \& Arikan, O. \& Centin, E. (1999). Robust adaptive fintering algorithms for $\alpha$-stable random process, IEEE Transactions Circuits Systems-II: Analog Digital Signal Processing, Vol. 46, No. 2, pp. 198-202, 1999.

Akhtar, M. T. \& Abe, M. \& Kawamata, M. (2005). A new structure for feedforward active noise control systems with improved online secondary path modeling. IEEE Transactions Speech Audio Processing, Vol. 13, No. 5, 2005, pp. 1082-1088.

Akhtar, M. T. \& Abe, M. \& Kawamata, M. (2006). A new variable step size LMS algorithm-based method for improved online secondary path modelling in active noise control systems. IEEE Transactions Audio Speech Language Processing, Vol. 14, No. 2, 2006, pp. 720-726.

Akhtar, M. T. \& Abe, M. \& Kawamata, M. (2007). On active noise control systems with online acoustic feedback path modeling. IEEE Transactions Audio Speech Language Processing, Vol. 15, No. 3, 2007, pp. 593-600.

Akhtar, M. T. \& Mitsuhashi, W. (2009a). Improving performance of FxLMS algorithm for active noise control of impulsive noise. Journal of Sound and Vibration, Vol. 327, No. 3-5. 2009, pp. 647-656.

Akhtar, M. T. \& Mitsuhashi, W. (2009b). Robust adaptive algorithms for active noise control of impulse noise. Proceedings of IEEE International Conference Acoustic Speech Signal Processing (ICASSP), pp. 261-264, April 19-24, 2009.

Akhtar, M. T. \& Mitsuhashi, W. (2011). Improving performance of hybrid active noise control systems for uncorrelated narrowband disturbances. IEEE Transactions Audio Speech Language Processing, 2011, (in press).

Bao, C. \& Sas, P. \& Brussel, H. V. (1993a). Comparison of two online identification algorithms for active noise control. Proceedings of Recent Advances in Active Control of Sound Vibration, 1993, pp. 38Ü-51.

Bao, C. \& Sas, P. \& Brussel, H. V. (1993b). Adaptive active control of noise in 3-D reverberant enclosure. Journal of Sound and Vibration, Vol. 161, No. 3, Mar. 1993, pp. 501-514.

Bonito, G. \& Elliott, S. J. \& Boucher, C. C. (1997). Generation of zones of quiet using a virtual microphone arrangement. Journal of Acoustical Society of Amerrica, Vol. 101, No. 6, June 1997, pp. 3498-3516.

Bouchard, M. \& Quednau, S. (2000). Multichannel recursive-least-squares algorithms and fast-transversal-filter algorithms for active noise control and sound reproduction systems. IEEE Transactions Speech Audio Processing, Vol. 8, 2000, pp. 606-618.

Crawford, D. H. \& Stewart, R. W. (1997). Adaptive IIR filtered-v algorithms for active noise control. Journal of Acoustical Society of America, Vol. 101, No. 4, 1997, pp. 2097-2103.

Castañé-Selga, R. \& Sánchez Peña, R. S. (2010). Active noise hybrid time-varying control for motorcycle helmets. IEEE Transactions Control Systems Technology, Vol. 18, No. 3, May 2010, pp. 602-612.

Douglas, S. C. (1994). A family of normalized LMS algorithms. IEEE Signal Processing Letters, Vol. 1, No. 3, 1994, pp. 49-51. 
Elliott, S. J. \& Stothers, I. M. \& Nelson, P. A. (1987) A multiple error LMS algorithm and its application to the active control of sound and vibration. IEEE Transactions Acoustic Speech Signal Processing, Vol. ASSP-35, Oct. 1987, pp. 1423-1434.

Elliot, S. J. (2001). Signal Processing for Active Control, London, U.K.: Academic Press.

Eriksson, L. J.; Allie, M. C. \& Greiner, R. A. (1987). The selection and application of an IIR adaptive filter for use in active sound attenuation. IEEE Transactions Acoustic Speech Signal Processing, Vol. 35, No. 1, 1987, pp. 433-437.

Eriksson, L. J. \& Allie, M. C. (1989). Use of random noise for on-line transducer modeling in an adaptive active attenuation system. Journal of Acoustical Society of Amerrica, Vol. 85, No. 2, Feb. 1989, pp. 797-802.

Esmailzadeh, E. \& Alasty, A. \& Ohadi, A. R. (2002). Hybrid active noise control of a one-dimensional acosutic duct. Transactions of the ASME, Vol. 124, 2002, pp. 10-18.

Gan, W. S. \& Kuo, S. M. (2002). An integrated audio and active noise control headsets. IEEE Transactions Consumer Electronics, Vol. 48, No. 2, May 2002, pp. 242-247.

Guo, J. N. \& Pan, J. (2000). Active control of moving noise source: effects of off-axis source position. Journal of Sound and Vibration, Vol. 215, 2000, pp. 457-475.

Jeon, H. J. \& Chang, T. G. \& Kuo, S. M. (2010). Analysis of frequency mismatch in narrowband active noise control. IEEE Transactions Audio Speech Language Processing, Vol. 18, No. 6, Aug. 2010, pp. 1632-1642.

Kida, M. \& Hirayama, R. \& Kajikawa, Y. \& Tani, T. \& Kurumi, Y. (2009). Head-mounted active noise control system for MR noise. Procedings IEEE Internation Confernece Acoustic Speech Signal Processing, 2009, pp. 245-248.

Kondo, K. \& Nakagawa, K. (2007). Speech emission control using active cancellation. Speech Communication, Vol. 49, No. 9, September 2007, pp. 687-696.

Kuo, S. M. \& Ji, M. J. (1995). Development and analysis of an adaptive noise equalizer. IEEE Transactions Speech Audio Processing, Vol. 3, May 1995, pp. 217-222.

Kuo, S. M. \& Yang, Y. (1996). Broadband adaptive noise equalizer. IEEE Signal Processing Letters, Vol. 3, No. 8, August 1996, pp. 234-235.

Kuo, S. M. \& Ji, M. (1996). Passband disturbance reduction in periodic active noise control systems. IEEE Transactions Speech Audio Processing, Vol. 4, No. 2, 1996, pp. 96-103.

Kuo, S. M. \& Morgan, D. R. (1996). Active Noise Control Systems-Algorithms and DSP Implementations, New York: Wiley.

Kuo, S. M. \& Tahernezhadi, M. (1997). Frequency-domain periodic active noise control and equalization. IEEE Transactions Speech Audio Processing, Vol. 5, 1997, pp. 348-358.

Kuo, S. M. \& Vijayan, D. (1997). A secondary path modeling technique for active noise control systems. IEEE Transactions Speech Audio Processing, Vol. 5, No. 4, July 1997, pp. 374-377.

Kuo, S. M. \& Morgan, D. R. (1999). Active noise control: A tutorial review. Proceedings of IEEE, Vol. 87, June 1999, pp. 943-973.

Kuo, S. M. \& Gan, W. S. (2004). Active noise control systems with optimized secondary path. Proceedings IEEE International Conference Control Applications, pp. pp. 765-770, September 2004.

Kuo, S. M. \& Mitra, S. \& Gan, W. S. (2006). Active noise control system for headphone applications. IEEE Transactions on Control Systems Technology, Vol. 14, No. 2, March 2006, pp. 331-335. 
Kuo, S. M. \& Puvvala, A. B. (2006). Effects of frequency separation in periodic active noise control systems. IEEE Transactions Audio Speech Language Processing, Vol. 14, No. 5, Sept. 2006, pp. 1857-1866.

Kuo, S. M. \& Gireddy, R. (2007). Real-time experiment of snore active noise control. Proceedings of IEEE International Conference on Control Applications, 1-3 Oct. 2007, pp. 1342-1346.

Kuo, S. M. \& Chakravarthy, S. R. \& Gireddy, R. \& Gupta, A. (2008). Experiment of active snore noise control systems. Noise Control Engineering Journal, Vol. 56, No. 1, Jan-Feb., 2008, pp. 16-24.

Kuo, S. M. \& Kuo, K. \& Gan, W. S. (2010). Active noise control: Open problems and challenges. Proceedings of International Conference on Green Circuits and Systems (ICGCS), pp. 164-169, Shanghai, 21-23 June 2010.

Leahy, R. \& Zhou, Z. \& Hsu, Y. C. (1995). Adaptive filtering of stable processes for active attenuation of impulsive Noise. Proceedings of IEEE International Conference Acoustic Speech Signal Processing (ICASSP), Vol. 5, pp. 2983-2986, May 1995.

Liu, L. \& Gujjula, S. \& Thanigai, P. \& Kuo, S. M. (2008). Still in womb: intrauterine acoustic embedded active noise control for infant incubators. Advances in Acoustics and Vibration, Vol. 2008, Article ID 495317, 9 pages, doi:10.1155/2008/495317.

Lueg, P. (1936). Process of silencing sound oscillations. US Patent, 2043416, June 9, 1936.

Morgan, D. R. (1980). An analysis of multiple correlation cancellation loops with a filter in auxiliary path. IEEE Transactions Acoustic Speech Signal Processing, Vol. ASSP-28, Aug. 1980, pp. 454-467.

Nikias, C. L. (1995). Signal Processing with Alpha-stable Distribution and Applications, Wiley, New York.

Omoto, A. \& Morie, D. \& Fujuwara, K. (2002). Behavior of adaptive algorithms in active noise control systems with moving noise sources. Acoustical Science and Technology, Vol. 23, No. 2, 2002, pp. 84-89.

Park, Y. C. \& Sommerfeldt, S. D. (1996). A fast adaptive noise control algorithm based on lattice structure. Appl. Acoust., Vol. 47, No. 1, 1996, pp. 1-25.

Reddy, E. P. \& Das, D. P. \& Prabhu, K. M. M. (2008). Fast adaptive algorithms for active control of nonlinear noise processes. IEEE Transactions Signal Processing, Vol. 56, No. 9, Sep. 2008, pp. 4530-4536.

Saito, N. \& Sone, T. (1996). Influence of modeling error on noise reduction performance of active noise control systems using filtered-x LMS algorithm. Joural of Acoustical Society of Japan (E), Vol. 17, No. 4, Apr. 1996, pp. 195-202.

Shao, M. \& Nikias, C. L. (1993). Signal processing with frqactional lower order moments: Stable processes and their appllications. Proceedings of IEEE. Vol. 81, No. 7, 1993, pp. 986-1010.

Strauch, P. \& Mulgrew, B. (1998). Active control of nonlinear noise processes in a linear duct. IEEE Transactions Signal Processing, Vol. 46, No. 9, Sep. 1998, pp. 2404-2412.

Sun, X. \& Kuo, S. M. \& and Meng, G. (2006). Adaptive algorithm for active control of impulsive noise. Journal of Sound and Vibration, Vol. 291, No. 1-2. 2006, pp. 516-522.

Sun, X. \& Kuo, S. M. (2007). Active narrowband noise control systems usign cascading adaptive filters. IEEE Transactions Audio Speech Language Processing, Vol. 15, No. 2, 2007, pp. 586-592.

Widrow, B. \& Stearns, S. D. (1985). Adaptive Signal Processing, Prentice Hall, New Jersey. 
Xiao, Y. \& Ward, R. K. \& Ma, L. \& Ikuta, A. (2005).A new LMS-based Fourier analyzer in the presence of frequency mismatch and applications. IEEE Transactions Circuits and Systems-I: Regular Papers, Vol. 52, No. 1, Jan. 2005, pp. 230-245.

Xiao, Y. \& Ma, L. \& Khorasani, K. \& Ikuta, A. (2006). A new robust narrowband active noise control system in the presence of frequency mismatch. IEEE Transactions Audio Speech Language Processing, Vol. 14, No. 6, Nov. 2006, pp. 2189-2200.

Zhang, M. \& Lan, H. \& Ser, W. (2001). Cross-updated active noise control system with online secondary path modeling. IEEE Transactions Speech Audio Processing, Vol. 9, No. 5, July 2001, pp. 598-602. 


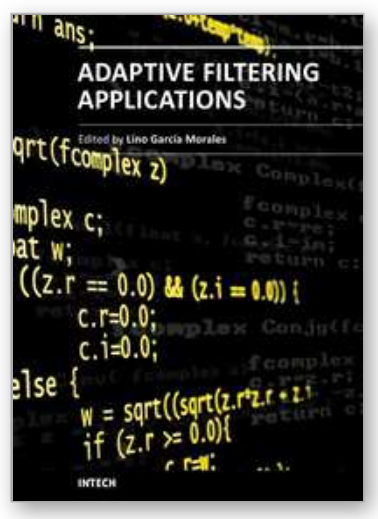

\author{
Adaptive Filtering Applications \\ Edited by Dr Lino Garcia
}

ISBN 978-953-307-306-4

Hard cover, 400 pages

Publisher InTech

Published online 24, June, 2011

Published in print edition June, 2011

Adaptive filtering is useful in any application where the signals or the modeled system vary over time. The configuration of the system and, in particular, the position where the adaptive processor is placed generate different areas or application fields such as: prediction, system identification and modeling, equalization, cancellation of interference, etc. which are very important in many disciplines such as control systems, communications, signal processing, acoustics, voice, sound and image, etc. The book consists of noise and echo cancellation, medical applications, communications systems and others hardly joined by their heterogeneity. Each application is a case study with rigor that shows weakness/strength of the method used, assesses its suitability and suggests new forms and areas of use. The problems are becoming increasingly complex and applications must be adapted to solve them. The adaptive filters have proven to be useful in these environments of multiple input/output, variant-time behaviors, and long and complex transfer functions effectively, but fundamentally they still have to evolve. This book is a demonstration of this and a small illustration of everything that is to come.

\title{
How to reference
}

In order to correctly reference this scholarly work, feel free to copy and paste the following:

Akhtar Muhammad Tahir, Mitsuhashi Wataru and Nishihara Akinori (2011). Applications of Adaptive Filtering: Recent Advancements in Active Noise Control, Adaptive Filtering Applications, Dr Lino Garcia (Ed.), ISBN: 978953-307-306-4, InTech, Available from: http://www.intechopen.com/books/adaptive-filteringapplications/applications-of-adaptive-filtering-recent-advancements-in-active-noise-control

\section{INTECH}

open science | open minds

\section{InTech Europe}

University Campus STeP Ri

Slavka Krautzeka 83/A

51000 Rijeka, Croatia

Phone: +385 (51) 770447

Fax: +385 (51) 686166

www.intechopen.com

\section{InTech China}

Unit 405, Office Block, Hotel Equatorial Shanghai

No.65, Yan An Road (West), Shanghai, 200040, China

中国上海市延安西路65号上海国际贵都大饭店办公楼405单元

Phone: +86-21-62489820

Fax: +86-21-62489821 
(C) 2011 The Author(s). Licensee IntechOpen. This chapter is distributed under the terms of the Creative Commons Attribution-NonCommercialShareAlike-3.0 License, which permits use, distribution and reproduction for non-commercial purposes, provided the original is properly cited and derivative works building on this content are distributed under the same license. 